\title{
Convexity, moduli of smoothness and a Jackson-type inequality
}

\author{
Z. Ditzian and A. Prymak
}

\begin{abstract}
For a Banach space $B$ of functions which satisfies for some $m>0$

$$
\max \left(\|F+G\|_{B},\|F-G\|_{B}\right) \geq\left(\|F\|_{B}^{s}+m\|G\|_{B}^{s}\right)^{1 / s}, \quad \forall F, G \in B
$$

a significant improvement for lower estimates of the moduli of smoothness $\omega^{r}(f, t)_{B}$ is achieved. As a result of these estimates, sharp Jackson inequalities which are superior to the classical Jackson type inequality are derived. Our investigation covers Banach spaces of functions on $\mathbb{R}^{d}$ or $\mathbb{T}^{d}$ for which translations are isometries or on $S^{d-1}$ for which rotations are isometries. Results for $C_{0}$ semigroups of contractions are derived. As applications of the technique used in this paper, many new theorems are deduced. An $L_{p}$ space with $1<p<\infty$ satisfies $(*)$ where $s=\max (p, 2)$, and many Orlicz spaces are shown to satisfy $(*)$ with appropriate $s$.
\end{abstract}

Key words and phrases: Moduli of smoothness, Jackson inequality, $L_{p}$ spaces, Orlicz spaces.

AMS subject classification: $\quad$ 41A63, 41A17, 41A25, 46B20, 47D60

\section{Introduction}

For a Banach space $B$ of functions on $\mathbb{R}^{d}$ or $\mathbb{T}^{d}$ for which translations are continuous isometries and whose norm satisfies for some $1<q \leq 2$ and some $M \geq 1$

$$
\frac{1}{2}\|F+G\|_{B}+\frac{1}{2}\|F-G\|_{B} \leq\left(\|F\|_{B}^{q}+M\|G\|_{B}^{q}\right)^{1 / q}, \forall F, G \in B
$$


the first author (see Di,88) derived a sharp version of the Marchaud inequality i.e. an estimate of the $r$-th modulus of smoothness $\omega^{r}(f, t)_{B}$ (see (1.5) below) by an expression involving $\omega^{r+1}(f, t)_{B}$, which implies a sharper version of the converse inequality (see also [To]). Analogous results were achieved for functions on the sphere (see Di,99). In the other direction, a sharp Jackson inequality and a sharp lower estimate of $\omega^{r}(f, t)_{L_{p}}$ for $1<p<\infty$ were given in [Da-Di-Ti] using a version of the Littlewood-Paley inequality. Here, we will use the following dual inequality to (1.1), given by

$$
\max \left(\|F+G\|_{B},\|F-G\|_{B}\right) \geq\left(\|F\|_{B}^{s}+m\|G\|_{B}^{s}\right)^{1 / s}, \forall F, G \in B
$$

for some $2 \leq s<\infty$ and $m>0$, to obtain the sharp Jackson inequality and the lower estimate of $\omega^{r}(f, t)_{B}$. This includes the result for $L_{p}, \quad 1<p<\infty$, since for $B=L_{p}$ when $1<p<\infty$, (1.2) is satisfied with $s=\max (2, p)$. An important portion of the paper will be dedicated to the lower estimate of $\sup _{0<u \leq t}\left\|(T(u)-I)^{r} f\right\|_{B}$, where $T(u)$ is a $C_{0}$ semigroup of contractions, and to applications of the lower estimate in approximation theory. An example of such an application is the sharp Jackson inequality for polynomial approximation on a simplex with Jacobi weights using the $L_{p}$ norm where $1<p<\infty$ or some other Orlicz norm which satisfies (1.2).

The condition (1.2) depends on the particular norm of $B$ and may not be satisfied by an equivalent norm of $B$. For our results we will need a norm on $B$ which satisfies simultaneously (1.2) and the condition that $T(u)$ is a contraction on $B$ or that translation by $\xi$ is a contraction or an isometry on $B$, which also is not inherited by an equivalent norm. However, for the conclusion of our results any equivalent norm of $B$ will do. In short, we need the condition that $B$ possesses a norm for which $T(u)$ are contractions and which simultaneously satisfies (1.2); however, the results are valid for any equivalent norm on $B$.

The following theorem is perhaps typical of the results achieved in the present paper.

Theorem 1.1. Suppose $B$ is a Banach space of functions on $\mathbb{R}^{d}$ or $\mathbb{T}^{d}$ with a norm 
satisfying (1.2) for some $s, \quad 2 \leq s<\infty$ and

$$
\|f(\cdot+\xi)\|_{B}=\|f(\cdot)\|_{B}, \quad \lim _{|h| \rightarrow 0}\|f(\cdot+h)-f(\cdot)\|_{B}=0, \quad\|f(-\cdot)\|_{B}=\|f(\cdot)\|_{B}
$$

for any $f \in B$ and $\xi, h \in \mathbb{R}^{d}$. Then for $C$ independent of $f, t$ and $n$

$$
2^{-n r}\left\{\sum_{j=1}^{n} 2^{j r s} \omega^{r+1}\left(f, 2^{-j}\right)_{B}^{s}\right\}^{1 / s} \leq C \omega^{r}\left(f, 2^{-n}\right)_{B}
$$

where

$$
\omega^{r}(f, t)_{B}=\sup _{|h| \leq t}\left\|\Delta_{h}^{r} f\right\|_{B}, \Delta_{h} f(x)=f(x+h)-f(x) \quad \text { and } \quad \Delta_{h}^{\ell+1} f=\Delta_{h}\left(\Delta_{h}^{\ell} f\right) .
$$

The inequality (1.4) is sharper than the classical $\omega^{r+1}(f, t)_{B} \leq 2 \omega^{r}(f, t)_{B}$ and is shown in [Da-Di-Ti, Section 10] to be optimal for $L_{p}, \quad 1<p<\infty$.

Throughout this paper constants will be positive and may depend on the space $\left(B, C\left(\mathbb{R}^{d}\right), L_{p}\left(\mathbb{R}^{d}\right)\right.$ etc.) and on $r$ but will be valid for all the elements of the space and will be independent of $t, n, j$ and $\ell$. Furthermore, unless otherwise specified, when a condition, result, or estimate is given in a theorem, definition, or remark concerning functions in some space, it applies to all the functions in that space.

\section{The basic inequality}

In this section we derive the basic inequality used throughout this paper.

Theorem 2.1. Suppose that $B$ is a Banach space of functions, that $T: B \rightarrow B$ is a linear contraction operator, that is $\|T f\|_{B} \leq\|f\|_{B}$ and suppose also that (1.2) is satisfied with a given $s, \quad 2 \leq s<\infty$ and $m>0$. Then for some $m_{1}>0$

$$
\left\|\Delta_{T}^{r} f\right\|_{B} \geq m_{1}\left(\sum_{j=0}^{\infty} 2^{-j r s}\left\|\Delta_{T^{2}}^{r+1} f\right\|_{B}^{s}\right)^{1 / s}
$$

where

$$
\Delta_{T^{2}} f=T^{2^{\ell}} f-f \quad \text { and } \quad \Delta_{T^{2^{\ell}}}^{k+1} f=\Delta_{T^{2^{\ell}}}\left(\Delta_{T^{2}}^{k} f\right) .
$$


Remark 2.2. Examples of such Banach spaces on $\mathbb{R}^{d}, \mathbb{T}^{d}$ or $S^{d-1}$ are $L_{p}$ spaces for $1<p<\infty$ where $s=\max (p, 2)$. An example of $T$ on a space of functions on $\mathbb{R}^{d}$ (and $\left.\mathbb{T}^{d}\right)$ is $T f(x)=f(x+\xi)$ with $x, \xi \in \mathbb{R}^{d}$. An example of $T$ on a space of functions on $S^{d-1}$ is $T f(x)=f(\rho x)$ with $x \in S^{d-1}$ and $\rho \in S O(d)$ (the orthogonal matrices on $\mathbb{R}^{d}$ whose determinant equals 1). Also $T=T(t)$ may be a semigroup of contractions, the simplest being $T(t) f(x)=f(x+t)$ on $L_{p}\left(\mathbb{R}_{+}\right)$, but other examples important for applications will be described at length.

Proof. Let $\widetilde{T}$ be any linear contraction operator on $B$. We note that $\widetilde{T}^{n} f=\widetilde{T}\left(\widetilde{T}^{n-1} f\right)$ and follow Di,88 to define $F=\frac{1}{2}\left(\widetilde{T}^{2}-I\right) \varphi$ and $G=-\frac{1}{2}(\widetilde{T}-I)^{2} \varphi, \varphi \in B$, so that $F+G=(\widetilde{T}-I) \varphi$ and $F-G=\widetilde{T}(\widetilde{T}-I) \varphi$. As $\widetilde{T}$ is a contraction, we have $\max (\| F+$ $\left.G\left\|_{B},\right\| F-G \|_{B}\right)=\|F+G\|_{B}=\|(\widetilde{T}-I) \varphi\|_{B}$ and by (1.2) with $\varphi=(\widetilde{T}-I)^{r-1} f, f \in B$, we obtain

$$
\left\|(\widetilde{T}-I)^{r} f\right\|_{B}^{s} \geq \frac{1}{2^{s}}\left\|\left(\widetilde{T}^{2}-I\right)(\widetilde{T}-I)^{r-1} f\right\|_{B}^{s}+m \frac{1}{2^{s}}\left\|(\widetilde{T}-I)^{r+1} f\right\|_{B}^{s} .
$$

Recalling that $\widetilde{T}$ is a contraction, we have $\left\|\left(\widetilde{T}^{2}-I\right)^{r} f\right\|_{B}^{s}=\left\|(\widetilde{T}+I)^{r}(\widetilde{T}-I)^{r} f\right\|_{B}^{s} \leq 2^{(r-1) s}\left\|(\widetilde{T}+I)(\widetilde{T}-I)^{r} f\right\|_{B}^{s}=2^{(r-1) s}\left\|\left(\widetilde{T}^{2}-I\right)(\widetilde{T}-I)^{r-1} f\right\|_{B}^{s}$, which, combined with (2.3), yields

$$
\left\|(\widetilde{T}-I)^{r} f\right\|_{B}^{s} \geq \frac{1}{2^{r s}}\left\|\left(\widetilde{T}^{2}-I\right)^{r} f\right\|_{B}^{s}+m \frac{1}{2^{s}}\left\|(\widetilde{T}-I)^{r+1} f\right\|_{B}^{s} .
$$

Now we use (2.4) iteratively with $\widetilde{T}=T, \widetilde{T}=T^{2}, \widetilde{T}=T^{4}, \ldots, \widetilde{T}=T^{2^{\ell}}$ to obtain

$$
\begin{aligned}
\left\|(T-I)^{r} f\right\|_{B}^{s} & \geq \frac{1}{2^{r s}}\left\|\left(T^{2}-I\right)^{r} f\right\|_{B}^{s}+m \frac{1}{2^{s}}\left\|(T-I)^{r+1} f\right\|_{B}^{s} \\
& \geq \frac{1}{2^{2 r s}}\left\|\left(T^{4}-I\right)^{r} f\right\|_{B}^{s}+m \frac{1}{2^{s}}\left(\left\|(T-I)^{r+1} f\right\|_{B}^{s}+\frac{1}{2^{r s}}\left\|\left(T^{2}-I\right)^{r+1} f\right\|_{B}^{s}\right) \\
& \geq \ldots \geq \frac{1}{2^{(\ell+1) r s}}\left\|\left(T^{2^{\ell+1}}-I\right)^{r} f\right\|_{B}^{s}+m \frac{1}{2^{s}}\left(\sum_{j=0}^{\ell} \frac{1}{2^{r s j}}\left\|\left(T^{2^{j}}-I\right)^{r+1} f\right\|_{B}^{s}\right) \\
& \geq\left(\frac{m}{2^{s}}\right) \sum_{j=0}^{\ell} \frac{1}{2^{r s j}}\left\|\left(T^{2^{j}}-I\right)^{r+1} f\right\|_{B}^{s},
\end{aligned}
$$


which implies (2.1) with $m_{1}=\frac{m^{1 / s}}{2}$.

The inequality (2.1), which is at the core of most of the results in this paper, is very simple, but to apply it successfully, we will need many and perhaps more sophisticated results.

\section{The condition on the space}

In this section we will discuss the condition (1.2), exhibit spaces for which it is valid and for what $s$. The condition (1.1) was shown in [Di,88] to be equivalent to the condition

$$
\eta_{B}(\sigma)=\sup _{\substack{\|E\|=1 \\\|G\|=\sigma}}\left(\frac{1}{2}\|F+G\|_{B}+\frac{1}{2}\|F-G\|_{B}-1\right), \quad \eta_{B}(\sigma) \leq k \sigma^{q},
$$

which was extensively investigated, and spaces $B$ satisfying (3.1) are described (see [Li-Tz, p.63]) as having modulus of smoothness of power type $q$. We note that the concept modulus of smoothness in [Li-Tz] describes the smoothness of the unit ball of the Banach space $B$ (in relation to a specific norm), and is not related to the concept with the same name (see for instance (1.5) ) in approximation theory describing smoothness of a function (i.e. an element of $B$ ). We note that we found (1.1) easier to use in classical analysis and also easier to verify (see [De-Lo, p.49]).

In the next theorem we show that (1.2) is dual to (1.1), and use that later to examine spaces that satisfy (1.2) and for what $s$. As a result we will show (later) that a big class of Orlicz spaces satisfies (1.2) and give examples of such spaces.

Theorem 3.1. Suppose $B$ is a Banach space endowed with a norm which for some $q$, $1<q \leq 2$ satisfies

$$
\frac{1}{2}\|x+y\|_{B}+\frac{1}{2}\|x-y\|_{B} \leq\left(\|x\|_{B}^{q}+M\|y\|_{B}^{q}\right)^{1 / q} \quad \text { for all } \quad x, y \in B .
$$

Then the dual of $B, \quad X=B^{*}$ (with the norm dual to that satisfying (3.2)) satisfies

$$
\max \left(\|\varphi+\psi\|_{X},\|\varphi-\psi\|_{X}\right) \geq\left(\|\varphi\|_{X}^{s}+m\|\psi\|_{X}^{s}\right)^{1 / s} \quad \text { for all } \varphi, \psi \in X=B^{*}
$$


with $s=\frac{q}{q-1}\left(\frac{1}{s}+\frac{1}{q}=1\right)$ and $m=M^{-1 /(q-1)}$. Moreover, if for a given norm of $X$ (3.3) is satisfied, then $B=X^{*}$ (with norm dual to that satisfying (3.3)) satisfies (3.2).

Proof. Define the operator $A$ on $(x, y) \in B \times B=\widetilde{B}$ by

$$
A(x, y)=\left(\frac{x+y}{2}, \frac{x-y}{2}\right)
$$

which we consider as a transformation between $\widetilde{B}$ with the norm $\|(u, v)\|_{\widetilde{B}_{1}}=\|u\|_{B}+\|v\|_{B}$ and $\widetilde{B}$ with the (equivalent) norm $\|(u, v)\|_{\widetilde{B}_{2}}=\left(\|u\|_{B}^{q}+M\|v\|_{B}^{q}\right)^{1 / q}$.

Using (3.2), we now have $\|A\|_{\widetilde{B}_{2} \rightarrow \widetilde{B}_{1}} \leq 1$. The dual to $\widetilde{B}, \widetilde{B}^{*}$, is given by $(\varphi, \psi)(u, v)=$ $\varphi u+\psi v$ where $\varphi, \psi \in B^{*}$. To calculate $A^{*}$, we write

$$
(\bar{\varphi}, \bar{\psi}) A(x, y)=\frac{1}{2}(\bar{\varphi}, \bar{\psi})(x+y, x-y)=\frac{1}{2}(\bar{\varphi}+\bar{\psi}, \bar{\varphi}-\bar{\psi})(x, y)=A^{*}(\bar{\varphi}, \bar{\psi})(x, y)
$$

Setting $\frac{\bar{\varphi}+\bar{\psi}}{2}=\varphi, \quad \frac{\bar{\varphi}-\bar{\psi}}{2}=\psi$, we have $A^{*}(\varphi+\psi, \varphi-\psi)=(\varphi, \psi)$.

Since $\|A\|_{\widetilde{B}_{2} \rightarrow \widetilde{B}_{1}} \leq 1$, we have $\left\|A^{*}\right\|_{\widetilde{B}_{1}^{*} \rightarrow \widetilde{B}_{2}^{*}} \leq 1$. We now write

$$
\|(\varphi, \psi)\|_{\widetilde{B}_{1}^{*}}=\sup _{\|u\|_{B}+\|v\|_{B}=1}|(\varphi u+\psi v)| \leq \max \left(\|\varphi\|_{B^{*}},\|\psi\|_{B^{*}}\right),
$$

and equality follows, choosing $v=0$ if $\|\varphi\|_{B^{*}} \geq\|\psi\|_{B^{*}}$ and choosing $u=0$ otherwise.

For the norm of $\widetilde{B}_{2}^{*}$

$$
\begin{aligned}
\|(\varphi, \psi)\|_{\widetilde{B}_{2}^{*}} & =\sup \left\{|\varphi u+\psi v|:\left(\|u\|_{B}^{q}+M\|v\|_{B}^{q}\right)^{1 / q}=1\right\} \\
& \leq \sup \left\{\|\varphi\|_{B^{*}}\|u\|_{B}+\left\|M^{-1 / q} \psi\right\|_{B^{*}}\left\|M^{1 / q} v\right\|_{B} ;\|u\|_{B}^{q}+M\|v\|_{B}^{q}=1\right\} \\
& \leq\left(\|\varphi\|_{B^{*}}^{s}+M^{-s / q}\|\psi\|_{B^{*}}^{s}\right)^{1 / s} \\
& =\left(\|\varphi\|_{B^{*}}^{s}+m\|\psi\|_{B^{*}}^{s}\right)^{1 / s}
\end{aligned}
$$

with $s=\frac{q}{q-1}$ and $m=M^{-1 /(q-1)}$. To show equality, we choose $a \geq 0$ and $b \geq 0$ for which $a^{q}+M b^{q}=1$ and $\left(a^{q}, M b^{q}\right)$ is proportional to $\left(\|\varphi\|_{B^{*}}^{s}, m\|\psi\|_{B^{*}}^{s}\right)$, and then choose $\left\|u_{n}\right\|_{B}=a, \quad\left\|v_{n}\right\|_{B}=b$ such that $\varphi u_{n} \rightarrow a\|\varphi\|_{B^{*}}$ and $\psi v_{n} \rightarrow b\|\psi\|_{B^{*}}$.

The second assertion can be obtained in a similar way using the operator $O$ on $(x, y) \in$ $X \times X=\widetilde{X}$ given by

$$
O(x, y)=(x+y, x-y)
$$


and endowing $\widetilde{X}$ with the norms $\|(x, y)\|_{\widetilde{X}_{1}}=\max \left(\|x\|_{X},\|y\|_{X}\right)$ and $\|(x, y)\|_{\widetilde{X}_{2}}=\left(\|x\|_{X}^{s}+\right.$ $\left.m\|y\|_{X}^{s}\right)^{1 / s}$, and hence $(2.2)$ is satisfied by all $x, y \in B_{1}=X^{*}$ and the $B_{1}$ norm. In the terminology of [Li-Tz, p.59] this implies that $B_{1}$ is uniformly smooth and hence (see [Li-Tz, p.61, Prop. 1.e.2(ii)]) $X$ is uniformly convex. We note that (3.2) and the above now imply (see [Li-Tz, p.61, Prop. 1.e.3]) that both $B$ and $X$ are reflexive. Therefore, $B_{1}=B$.

As a corollary of Theorem 3.1 we show that the condition (1.2) is satisfied by $L_{p}$ spaces.

Corollary 3.2. For $L_{p}$ with $1<p<\infty$

$$
\max \left(\|F+G\|_{p},\|F-G\|_{p}\right) \geq\left(\|F\|_{p}^{\max (p, 2)}+m\|G\|_{p}^{\max (p, 2)}\right)^{1 / \max (p, 2)}
$$

for some $m>0$.

Proof. We recall that for $L_{p}, \quad 1<p<\infty$, (3.2) is valid with $q=\min (p, 2)$ (see Di,88) and use Theorem 3.1 .

Remark 3.3. As (3.1) with $x=F$ and $y=G$ was shown to be equivalent to (3.2) (see Di,88) and (3.3) was shown to be dual to (3.2), the condition

$$
\begin{gathered}
\delta_{X}(\varepsilon) \geq K \varepsilon^{s} \quad \text { where } \\
\delta_{X}(\varepsilon) \equiv \inf \left(1-\|\varphi+\psi\|_{X} / 2: \varphi, \psi \in X,\|\varphi\|_{X}=\|\psi\|_{X}=1,\|\varphi-\psi\|_{X}=\varepsilon\right)
\end{gathered}
$$

which is dual to (3.1) (see [Li-Tz, p.63]) is equivalent to (3.3). Hence we note that the condition (3.3) on (a given norm of) a Banach space $X$ means that $X$ has a modulus of convexity of at least power type $s$ (see [Li-Tz, p.63]).

Remark 3.4. For a space $B$ both the inequalities (1.1) and (1.3) depend on the norm and may not be valid for an equivalent norm. However, the sharp Marchaud inequality or sharp converse inequality is valid if it is valid for an equivalent norm. It will be evident that the validity of the sharp Jackson inequality and of the lower estimate for the modulus of smoothness will, in the situations proved in this paper for one norm of $B$, imply their validity for any equivalent norm. 
Remark 3.5. On the face of it, it may seem that in Theorem 3.1 we neglected to treat the situation when $q>2$. However, as (3.2) is equivalent to $\eta_{B}(\sigma) \leq k \sigma^{q}$ (with $\eta_{B}(\sigma)$ of (3.1) ), and as $\eta_{B}(\sigma) / \sigma^{2}$ is equivalent to a non-increasing function for any Banach space (see [Li-Tz, p.64, Prop. 1.e.5]), a nontrivial Banach space (different from $\mathbb{R}$ or $\{0\}$ ) for which (3.2) is satisfied with $q>2$ does not exist.

We outline now the basic notations (and some facts) concerning Orlicz spaces (see [Ra-Re] and [Be-Sh, pp.265-280]) which we will use in this section and later. A Young function $\Phi$ is an increasing convex function on $\mathbb{R}_{+}$satisfying $\Phi(0)=0$. For a domain $\Omega$ and a (positive) measure $d \mu(x)$ the Orlicz class $\mathcal{M}(\Phi)$ and the Orlicz functional $M_{\Phi}(f)$ are given by

$$
\mathcal{M}(\Phi) \equiv\left\{f: M_{\Phi}(f) \equiv \int_{\Omega} \Phi(|f(x)|) d \mu(x)<\infty\right\}
$$

The Luxemburg norm of the Orlicz space is given by

$$
\|f\|_{O_{L}(\Phi)} \equiv \inf \left(a>0: M_{\Phi}\left(\frac{|f|}{a}\right) \leq 1\right) .
$$

$\Psi(y): \mathbb{R}_{+} \rightarrow \mathbb{R}_{+}$is the complementary Young function to $\Phi(x)$ satisfying $\lim _{x \rightarrow \infty} \frac{\Phi(x)}{x}=\infty$ if

$$
\Psi(y)=\sup \{x y-\Phi(x): x \geq 0\}, \quad y \geq 0 .
$$

The Orlicz norm of the Orlicz space is given by

$$
\|f\|_{O(\Phi)} \equiv \sup \left\{\int_{\Omega}|f(x) g(x)| d \mu(x): \int_{\Omega} \Psi(|g(x)|) d \mu(x) \leq 1\right\} .
$$

A Young function $\Phi$ satisfies the $\Delta_{2}$ condition if for some $K>0$

$$
\Phi(2 x) \leq K \Phi(x) \quad \text { for } \quad x \geq x_{0} \geq 0 \quad\left(x_{0}=0 \quad \text { when } \quad \mu(\Omega)<\infty\right)
$$

A Young function $\Psi$ satisfies the $\nabla_{2}$ condition if for some $a>1$

$$
\Psi(x) \leq \frac{1}{2 a} \Psi(a x) \quad \text { for } \quad x \geq x_{0} \geq 0 \quad\left(x_{0}=0 \quad \text { when } \quad \mu(\Omega)<\infty\right) .
$$


It is known that if $\Phi$ is a Young function, $\Psi$ given by (3.8) is a Young function as well. Also $\|f\|_{O_{L}(\Phi)} \leq\|f\|_{O(\Phi)} \leq 2\|f\|_{O_{L}(\Phi)}$ (see [Be-Sh, Th.8.14, p.272]). Moreover, if $\Phi$ satisfies the $\Delta_{2}$ condition, the complementary Young function $\Psi$ satisfies the $\nabla_{2}$ condition (see [Ra-Re, Cor.4, p.26]).

Lemma 3.6. Suppose $\Phi$ is a Young function and that $\Phi\left(u^{1 / s}\right)$ is concave for some $1<s<$ $\infty$. Then $\Psi\left(t^{1 / q}\right)$ is convex where $\Psi$ is the complementary Young function and $\frac{1}{s}+\frac{1}{q}=1$.

Proof. Let $g(u) \equiv \Phi\left(u^{1 / s}\right), u \geq 0$. Then

$$
g(u)=\inf _{z \geq 0}\left(g_{+}^{\prime}(z)(u-z)+g(z)\right)
$$

and as $\lim _{u \rightarrow \infty} g(u)=+\infty$, for every $z \geq 0$ we have $g_{+}^{\prime}(z)>0$. In other words, $g(u)=$ $\inf _{(a, b) \in L}(a u+b)$, where $L$ is a subset of $(0,+\infty) \times \mathbb{R}$. Therefore, $\Phi(x)=\inf _{(a, b) \in L}\left(a x^{s}+b\right)$. By the definition of $\Psi$,

$$
\Psi(y)=\sup _{x \geq 0}(x y-\Phi(x))=\sup _{x \geq 0} \sup _{(a, b) \in L}\left(x y-a x^{s}-b\right)=\sup _{(a, b) \in L} \sup _{x \geq 0}\left(x y-a x^{s}-b\right) .
$$

As $s>1$, the second supremum is achieved at $x=\left(\frac{y}{a s}\right)^{\frac{1}{s-1}}$. Hence,

$$
\Psi(y)=\sup _{(a, b) \in L}\left(\left(\frac{1}{(a s)^{\frac{1}{s-1}}}-a(a s)^{-q}\right) y^{q}-b\right)
$$

which means that $\Psi\left(t^{1 / q}\right)$ is a supremum of a family of functions linear in $t$, and therefore $\Psi\left(t^{1 / q}\right)$ is convex.

Lemma 3.7. Suppose $\Phi\left(u^{1 / s}\right)$ is concave for some $s, 2 \leq s<\infty$, where $\Phi$ is a Young function satisfying the $\nabla_{2}$ condition. Then there exist constants $A, m>0$ and a Young function $\widetilde{\Phi}(u)$, such that $A^{-1} \Phi(u) \leq \widetilde{\Phi}(u) \leq A \Phi(u)$, satisfying

$$
\max \left\{\|f+g\|_{O(\widetilde{\Phi})},\|f-g\|_{O(\widetilde{\Phi})}\right\} \geq\left(\|f\|_{O(\widetilde{\Phi})}^{s}+m\|g\|_{O(\widetilde{\Phi})}^{s}\right)^{1 / s}, \quad \text { for all } f, g \in \mathcal{M}(\Phi)
$$


Proof. The complementary Young function $\Psi$ satisfies the $\Delta_{2}$ condition, and $\Psi\left(t^{1 / q}\right)$ is convex for $\frac{1}{q}+\frac{1}{s}=1$ by the previous lemma. Thus, we can apply Lemma 2.2 of [Di-Pr] for $B=O_{L}(\Psi)$ and $M=\Psi$, to find a Young function $N=\widetilde{\Psi}$, equivalent to $\Psi$ such that

$$
\frac{\|f+g\|_{O_{L}(\widetilde{\Psi})}+\|f-g\|_{O_{L}(\widetilde{\Psi})}}{2} \leq\left(\|f\|_{O_{L}(\widetilde{\Psi})}^{q}+L\|g\|_{O_{L}(\widetilde{\Psi})}^{q}\right)^{1 / q}, \quad \text { for all } f, g \in \mathcal{M}(\Psi)
$$

with $L>0$. Let $\widetilde{\Phi}$ be the complementary Young function of $\widetilde{\Psi}$. The Young function $\widetilde{\Phi}$ is equivalent to $\Phi$ ([Ra-Re, Prop.2, p.15]) and the dual of $O_{L}(\widetilde{\Psi})$ is isometric to $O(\widetilde{\Phi})$ ([Ra-Re, Cor.9, p.111]). Hence, using Theorem 3.1, (3.11) implies (3.10).

Now we will show examples of Young functions $\Phi$ for which there exists an equivalent Young function $\widetilde{\Phi}$ such that $\widetilde{\Phi}\left(u^{1 / s}\right)$ is concave for some $s, 2 \leq s<\infty$, and which satisfies the $\nabla_{2}$ condition (consequently, the corresponding Orlicz spaces will satisfy (1.2)).

We intend to consider $\Phi(u)=u^{r}(1+|\ln u|)$ and $\Phi(u)=\max \left\{u^{\alpha}, u^{\beta}\right\}$ for appropriate values of $r, \alpha, \beta$. Note that these functions themselves (being convex) cannot satisfy the condition that $g(u) \equiv \Phi\left(u^{1 / s}\right)$ is concave for the following reason: $\Phi_{+}^{\prime}(1)>\Phi_{-}^{\prime}(1)$, and hence $g_{+}^{\prime}(1)>g_{-}^{\prime}(1)$. However, with proper $s, g$ can be concave near 0 and near $\infty$. Our task is to "patch" these pieces together to construct an equivalent function $\widetilde{\Phi}$ satisfying the necessary conditions.

Lemma 3.8. Let $\Phi$ be a Young function such that

$$
\Phi\left(u^{1 / s}\right) \text { is concave on }[0, a] \text { and on }[b, \infty),
$$

where $0<a<b, s \geq 2$. Then there is a Young function $\widetilde{\Phi}$ satisfying

$$
\widetilde{\Phi}(u)=c_{1} \Phi(u), \quad u \in[0, a],
$$

and

$$
\widetilde{\Phi}(u)=c_{2}+\Phi(u), \quad u \in[b, \infty),
$$

with some constants $c_{1}>0$ and $c_{2}$, which is equivalent to $\Phi(u)$ and also $\widetilde{\Phi}\left(u^{1 / s}\right)$ is concave on $[0, \infty)$. 
Proof. As $\Phi$ is convex, it is absolutely continuous and $\Phi^{\prime}$ exists almost everywhere and is non-decreasing. We choose $c_{1}$ to satisfy

$$
c_{1} \Phi^{\prime}(a-) a^{\frac{1}{s}-1}=\Phi^{\prime}(b+) b^{\frac{1}{s}-1} .
$$

We now define

$$
\phi(u):= \begin{cases}c_{1} \Phi^{\prime}(u), & u \in[0, a), \\ u^{1-\frac{1}{s}} \Phi^{\prime}(b+) b^{\frac{1}{s}-1}, & u \in[a, b], \\ \Phi^{\prime}(u), & u \in(b, \infty),\end{cases}
$$

and $\widetilde{\Phi}(x):=\int_{0}^{x} \phi(u) d u$. Clearly, (3.13) and (3.14) are satisfied. Also, as $\phi(a)=c_{1} \Phi^{\prime}(a-)$, $\phi(b)=\Phi^{\prime}(b+)$ and $\phi$ is increasing on $[a, b], \widetilde{\Phi}$ is a Young function. For $u \in[a, b]$ we obtain

$$
\left(\widetilde{\Phi}\left(u^{1 / s}\right)\right)^{\prime}=\frac{1}{s} \phi(u) u^{\frac{1}{s}-1}=\frac{1}{s} \Phi^{\prime}(b+) b^{\frac{1}{s}-1}=\text { const }=\left.\left(\widetilde{\Phi}\left(u^{1 / s}\right)\right)^{\prime}\right|_{u=a-}=\left.\left(\widetilde{\Phi}\left(u^{1 / s}\right)\right)^{\prime}\right|_{u=b+} .
$$

Hence, $\left(\widetilde{\Phi}\left(u^{1 / s}\right)\right)^{\prime}$ is non-increasing on $[0, \infty)$.

We observe that the resulting Young function $\widetilde{\Phi}$ is equivalent to $\Phi$.

Example 3.9. Let $\Phi(u)=\max \left\{u^{\alpha}, u^{\beta}\right\}$, where $1<\alpha<\beta$. Then $\Phi\left(u^{1 / s}\right)$ satisfies (3.12) for any $s \geq \max \{2, \beta\}$.

Proof. We have

$$
\Phi\left(u^{1 / s}\right)= \begin{cases}u^{\alpha / s}, & u \leq 1 \\ u^{\beta / s}, & u>1\end{cases}
$$

so both $\alpha / s$ and $\beta / s$ must not exceed 1 .

Example 3.10. Let $\Phi(u)=u^{r}(1+|\ln u|), r \geq(3+\sqrt{5}) / 2$ (which guarantees that $\Phi$ is a Young function). Then $\Phi\left(u^{1 / s}\right)$ satisfies (3.12) for any $s>r$ and does not satisfy (3.12) with $s=r$.

Proof. We have

$$
\Phi^{\prime}(u)= \begin{cases}u^{r-1}(r+1+r \ln u), & u>1 \\ u^{r-1}(r-1-r \ln u), & u<1\end{cases}
$$


and

$$
\Phi^{\prime \prime}(u)= \begin{cases}u^{r-2}\left(r^{2}+r-1+r(r-1) \ln u\right), & u>1, \\ u^{r-2}\left(r^{2}-3 r+1-r(r-1) \ln u\right), & u<1 .\end{cases}
$$

Hence, $r \geq(3+\sqrt{5}) / 2$ implies convexity of $\Phi$. We further compute

$$
\left(\Phi\left(u^{\frac{1}{s}}\right)\right)^{\prime}= \begin{cases}\frac{1}{s} u^{\frac{r}{s}-1}\left(r+1+\frac{r}{s} \ln u\right), & u>1, \\ \frac{1}{s} u^{\frac{r}{s}-1}\left(r-1-\frac{r}{s} \ln u\right), & u<1,\end{cases}
$$

and

$$
\left(\Phi\left(u^{\frac{1}{s}}\right)\right)^{\prime \prime}= \begin{cases}\frac{1}{s} u^{\frac{r}{s}-2}\left(\frac{r}{s}(r+2)-r-1+\frac{r}{s}\left(\frac{r}{s}-1\right) \ln u\right), & u>1, \\ \frac{1}{s} u^{\frac{r}{s}}-2\left(\frac{r}{s}(r-2)-r+1-\frac{r}{s}\left(\frac{r}{s}-1\right) \ln u\right), & u<1 .\end{cases}
$$

Under the condition $s>r$, the function $\left(\Phi\left(u^{\frac{1}{s}}\right)\right)^{\prime \prime}$ is clearly non-positive for $u<1$ and also non-positive for $u>u_{0}$, where $u_{0}$ is such that $\frac{r}{s}\left(\frac{r}{s}-1\right) \ln u_{0}=-1$. If $r=s$, then $\left(\Phi\left(u^{\frac{1}{s}}\right)\right)^{\prime \prime}=1$ for $u>1$.

Example 3.11. The Zygmund spaces $L_{p}(\log L)^{\alpha}$. Let $\Phi(u)=u^{p}(\ln (2+u))^{\alpha p}, \alpha p \geq 1$, $p \geq 1$ (see [Be-Sh, Def.6.11, p.252]). Then $\Phi\left(u^{1 / s}\right)$ satisfies (3.12) for any $s>p$ and does not satisfy (3.12) with $s=p$.

Proof. We find

$$
\Phi^{\prime}(u)=p u^{p-1} \ln ^{\alpha p-1}(2+u)\left(\ln (2+u)+\frac{\alpha u}{2+u}\right),
$$

and hence,

$$
\left(\Phi\left(u^{\frac{1}{s}}\right)\right)^{\prime}=\frac{p}{s} u^{\frac{p}{s}-1} \ln ^{\alpha p-1}\left(2+u^{\frac{1}{s}}\right)\left(\ln \left(2+u^{\frac{1}{s}}\right)+\frac{\alpha u^{\frac{1}{s}}}{2+u^{\frac{1}{s}}}\right) .
$$

Differentiating once more, we obtain

$$
\left(\Phi\left(u^{\frac{1}{s}}\right)\right)^{\prime \prime}=\frac{p}{s} u^{\frac{p}{s}-2} \ln ^{\alpha p-1}\left(2+u^{\frac{1}{s}}\right)\left(\ln \left(2+u^{\frac{1}{s}}\right)+\frac{\alpha u^{\frac{1}{s}}}{2+u^{\frac{1}{s}}}\right)\left(\frac{p}{s}-1+D(u)\right),
$$

where

$$
D(u)=\frac{u^{\frac{1}{s}}}{s\left(2+u^{\frac{1}{s}}\right)}\left(\frac{\alpha p-1}{\ln \left(2+u^{\frac{1}{s}}\right)}+\frac{1+\frac{2 \alpha}{2+u^{\frac{1}{s}}}}{\ln \left(2+u^{\frac{1}{s}}\right)+\frac{\alpha u^{\frac{1}{s}}}{2+u^{\frac{1}{s}}}}\right) .
$$


The sign of $\left(\Phi\left(u^{\frac{1}{s}}\right)\right)^{\prime \prime}$ for $u>0$ is determined by the sign of the last factor of the right hand side of (3.15). As $D(u)$ is positive for $u>0$, if $s=p$, then $\left(\Phi\left(u^{\frac{1}{s}}\right)\right)^{\prime \prime}>0$. If $s>p$, then $\frac{p}{s}-1<0$ and we can choose $a, b$ to satisfy (3.12) since

$$
\lim _{u \rightarrow 0+} D(u)=0 \quad \text { and } \quad \lim _{u \rightarrow \infty} D(u)=0 .
$$

Note that in all the above examples it is easy to verify that $\Phi$ satisfies the $\Delta_{2}$ and the $\nabla_{2}$ conditions.

\section{Applications using Holomorphic semigroups}

The operators $T(t), T(t): B \rightarrow B$ for $t \in[0, \infty)=\mathbb{R}_{+}$form a $C_{0}$ semigroup if $T(t+s) f=T(t) T(s) f$ and $\lim _{t \rightarrow 0+}\|T(t) f-f\|_{B}=0 . \quad\{T(t)\}_{t \geq 0}$ is a semigroup of contractions if $\|T(t) f\|_{B} \leq\|f\|_{B}$ for all $t \in \mathbb{R}_{+}$and $f \in B$. The infinitesimal generator $\mathcal{A}$ related to the semigroup $\{T(t)\}_{t \geq 0}$ is given by

$$
\mathcal{A} f \equiv \mathrm{s}-\lim _{t \rightarrow 0+} \frac{T(t) f-f}{t},
$$

(where $\underset{s-\lim _{t \rightarrow 0+}}{ } g_{t}=\varphi$ if $\left\|g_{t}-\varphi\right\|_{B} \rightarrow 0$ as $t \rightarrow 0+$ ) and the domain of $\mathcal{A}, \mathcal{D}(\mathcal{A}$ ), consists of all $f$ such that the limit in (4.1) exists. A holomorphic semigroup is a semigroup satisfying

$$
T(t) f \in \mathcal{D}(\mathcal{A}) \quad \text { for } \quad t>0 \quad \text { and } \quad t\|\mathcal{A} T(t) f\|_{B} \leq N\|f\|_{B}
$$

with $N$ independent of $t$ and $f$. (Note that (4.2) is essentially a Bernstein-type inequality.)

It was proved (see [Di-Iv, Th.5.1, p.74]) that for a holomorphic $C_{0}$ semigroup of contractions we have

$$
\left\|(T(t)-I)^{r} f\right\|_{B} \approx \inf _{g \in \mathcal{D}\left(\mathcal{A}^{r}\right)}\left(\|f-g\|_{B}+t^{r}\left\|\mathcal{A}^{r} g\right\|_{B}\right) \equiv K_{\mathcal{A}^{r}}\left(f, t^{r}\right)_{B}
$$


which is a strong converse inequality of type $A$ in the terminology of [Di-Iv]. We recall that by $A(t) \approx E(t)$ one means $C^{-1} A(t) \leq E(t) \leq C A(t)$. Using (4.3) and general properties of $K$-functionals, we have for holomorphic semigroups

$$
\left\|(T(t)-I)^{r} f\right\|_{B} \approx \sup _{0<u \leq t}\left\|(T(u)-I)^{r} f\right\|_{B} .
$$

As a corollary of Theorem 2.1 and (4.3) we obtain the following result.

Theorem 4.1. Suppose that $\{T(t)\}_{t \geq 0}$ is a holomorphic $C_{0}$ semigroup of contractions on a Banach space $B$ and that $B$ satisfies the condition (1.2) for some $2 \leq s<\infty$ and $m>0$. Then for any integer $r$

$$
K_{\mathcal{A}^{r}}\left(f, t^{r}\right)_{B} \geq C\left\{\sum_{j=1}^{\infty} 2^{-j r s} K_{\mathcal{A}^{r+1}}\left(f, 2^{j(r+1)} t^{r+1}\right)_{B}^{s}\right\}^{1 / s} .
$$

Proof. We use (2.1) with $T=T(t)$ and $T^{2^{\ell}}=T\left(t 2^{\ell}\right)$, to which we apply (4.3) (for both $r$ and $r+1)$, which yields $\left\|(T(t)-I)^{r} f\right\|_{B} \leq C_{1} K_{\mathcal{A}^{r}}\left(f, t^{r}\right)_{B}$ and $\left\|\left(T\left(t 2^{j}\right)-I\right)^{r+1} f\right\|_{B} \geq$ $C_{2} K_{\mathcal{A}^{r}}\left(f, 2^{j(r+1)} t^{r+1}\right)_{B}$ to complete the proof of (4.5).

The usefulness of Theorem 4.1 is clearly demonstrated by applying it to the GaussWeierstrass semigroup of operators (see for instance [Bu-Be, p.261]) given by

$$
W(t) f(x) \equiv \frac{1}{(4 \pi t)^{d / 2}} \int_{\mathbb{R}^{d}} \exp \left(\frac{-|x-u|^{2}}{4 t}\right) f(u) d u .
$$

Theorem 4.2. Suppose that B, a Banach space of functions on $\mathbb{R}^{d}$, satisfies (1.2) and (1.3) and that $B \subset \mathcal{S}^{\prime}$ which means that $B$ is continuously imbedded in the Schwartz space of tempered distribution. Then

$$
\begin{aligned}
\left\|(W(t)-I)^{r} f\right\|_{B} & \approx \sup _{u \leq t}\left\|(W(u)-I)^{r} f\right\|_{B} \approx \inf _{g \in \mathcal{D}\left(\Delta^{r}\right)}\left(\|f-g\|_{B}+t^{r}\left\|\Delta^{r} g\right\|_{B}\right) \\
& \equiv K_{\Delta^{r}}\left(f, t^{r}\right)_{B}, \\
K_{\Delta^{r}}\left(f, t^{r}\right)_{B} & \geq C\left\{\sum_{j=1}^{\infty} 2^{-j r s} K_{\Delta^{r+1}}\left(f, 2^{j(r+1)} t^{r+1}\right)_{B}^{s}\right\}^{1 / s}
\end{aligned}
$$


and

$$
K_{\Delta^{r}}\left(f, t^{r}\right)_{B} \geq C\left\{\sum_{j=1}^{\infty} 2^{-j r s} E_{2^{j / 2} t^{1 / 2}}(f)_{B}^{s}\right\}^{1 / s}
$$

where $\Delta$ is the Laplacian and $E_{\lambda}(f)_{B}$ is given by

$$
E_{\lambda}(f)_{B}=\inf \left\{\left\|f-\varphi_{\sigma}\right\|_{B}: \operatorname{supp} \widehat{\varphi}_{\sigma}(y) \subset(y:|y| \leq \lambda)\right\}
$$

where $\widehat{\varphi}_{\sigma}$ is the Fourier transform of $\varphi_{\sigma}$.

Proof. For $f \in B$ satisfying (1.3) we may use the Riemann vector valued integration in (4.6) to obtain for all $f \in B$

$$
\begin{gathered}
\|W(t) f\|_{B} \leq\|f\|_{B}, \quad \lim _{t \rightarrow 0+}\|W(t) f-f\|_{B}=0 \\
\Delta^{r} W(t) f \in B \quad \text { and } \quad\left\|\Delta^{r} W(t) f\right\|_{B} \leq\left(\frac{d}{t}\right)^{r}\|f\|_{B} .
\end{gathered}
$$

For $\varphi \in \mathcal{S}$, the Schwartz space of test functions, straightforward computation implies $\frac{W(t) \varphi-\varphi}{t}-\Delta \varphi \rightarrow 0$ in $\mathcal{S}$ and hence in $B^{*}$, the dual to $B$. Therefore, whenever $f \in \mathcal{D}(\Delta)$, that is when $\Delta f$ exists in the $\mathcal{S}^{\prime}$ sense and $\Delta f \in B$, we have

$$
\left\langle\frac{W(t) f-f}{t}-\Delta f, \varphi\right\rangle=\left\langle f, \frac{W(t) \varphi-\varphi}{t}-\Delta \varphi\right\rangle \rightarrow 0
$$

for all $\varphi \in \mathcal{S}$. For $f \in \mathcal{D}(\mathcal{A})$ with $\mathcal{A}$ the infinitesimal generator of $W(t)$ $\left\|\frac{W(t) f-f}{t}-\mathcal{A} f\right\|_{B} \rightarrow 0$ and $\left\|\frac{W(t+s) f-W(s) f}{t}-\mathcal{A} W(s) f\right\|_{B} \rightarrow 0$. As $W(s) f \in \mathcal{D}(\Delta)$ and $W(s) f \in \mathcal{D}(\mathcal{A})$, we have $\lim _{t \rightarrow 0+}\left\langle\frac{W(t+s) f-W(s) f}{t}-\Delta W(s) f, \varphi\right\rangle=0$ and $\lim _{t \rightarrow 0+}\left\langle\frac{W(t+s) f-W(s)}{t}-\right.$ $\mathcal{A} W(s) f, \varphi\rangle=0$ for all $\varphi \in \mathcal{S} \cap B^{*}$, and hence $\Delta W(s) f=\mathcal{A} W(s) f$ for all $s>0$.

For $f \in \mathcal{D}(\Delta)$ we can now write

$$
\|W(t+s) f-W(s) f\|_{B} \leq t\|\mathcal{A} W(s) f\|_{B}=t\|\Delta W(s) f\|_{B} \leq t\|\Delta f\|_{B} \quad \text { for all } \quad s>0
$$

and using (4.11), $\|W(t) f-f\|_{B} \leq t\|\Delta f\|_{B}$. 
Similarly, for $g \in \mathcal{D}\left(\Delta^{r}\right)$

$$
\left\|(W(t)-I)^{r} g\right\|_{B} \leq t^{r}\left\|\Delta^{r} g\right\|_{B} .
$$

The above directly implies the inequality $\left\|(W(t)-I)^{r} f\right\|_{B} \leq C K_{\Delta^{r}}\left(f, t^{r}\right)_{B}$. The proof of the inequality $K_{\Delta^{r}}\left(f, t^{r}\right)_{B} \leq C\left\|(W(t)-I)^{r} f\right\|_{B}$ follows exactly the proof of Theorem 5.1 in Di-Iv], replacing $\mathcal{A}$ by $\Delta$, which as it operates on $g=-\sum_{k=1}^{r}(-1)^{k}\left(\begin{array}{c}r \\ k\end{array}\right) T(k m t) f$, is the same.

For $B=L_{p}\left(\mathbb{R}^{d}\right), \quad 1 \leq p<\infty, \quad \Delta=\mathcal{A}$ (see the proof in [Bu-Be, Th.4.311, p.261]).

The inequality (4.8) now follows from Theorem 4.1 as (4.7) implies $K_{\mathcal{A}^{r}}\left(f, t^{r}\right)_{B} \approx$ $K_{\Delta^{r}}\left(f, t^{r}\right)_{B}$ for all $f, r$ and $t$. The inequality (4.9) follows from (4.8) and the inequality

$$
E_{\lambda}(f)_{B} \leq C K_{\Delta^{r}}\left(f, \lambda^{-2 r}\right)_{B}
$$

The inequality (4.12) was proved in [Da-Di,04, (2.9), p.271] for $B=L_{p}\left(\mathbb{R}^{d}\right), \quad 1 \leq p \leq \infty$. In fact, (4.12) follows for any $B$ satisfying (1.3), as all we need in the proof of [Da-Di,04, 271-272] is that the linear convolution operators $R_{\lambda, \ell, b} f$ there satisfy

$$
\left\|R_{\lambda, \ell, b} f\right\|_{B} \leq C_{1}\|f\|_{B} \quad \text { and } \quad\left\|R_{\lambda, \ell, b} g-g\right\|_{B} \leq C_{2} \frac{1}{\lambda^{2 \ell}}\left\|\Delta^{\ell} g\right\|_{B} .
$$

We define $F=f * \varphi$ with $\varphi \in B^{*}$ and $\|\varphi\|_{B^{*}}=1$ such that $\varphi$ satisfies

$$
\begin{aligned}
\left\|R_{\lambda, \ell, b} f\right\|_{B}-\varepsilon & \leq\left|R_{\lambda, \ell, b} F(0)\right| \leq \sup _{x}\left|R_{\lambda, \ell, b} F(x)\right| \\
& =\left\|R_{\lambda, \ell, b} F\right\|_{\infty} \leq C_{1}\|F\|_{\infty} \leq C_{1}\|f\|_{B} .
\end{aligned}
$$

Similarly, we obtain the second inequality of (4.13) using $G=g * \varphi$.

Remark 4.3. For $L_{p}\left(\mathbb{R}^{d}\right)$ a somewhat more general result than in Theorem 4.2 was proved in [Da-Di-Ti, Theorem 7.1] using a completely different method. Here the proof is much simpler and applies to a wide class of Orlicz spaces (see Section 3), and perhaps to other spaces that satisfy (1.2) with some norm of $B$ that satisfies (1.3) at the same time. Orlicz spaces described in Section 3 satisfy (1.2) with the same norm for which (1.3) is valid. 
Remark 4.4. Using the monotonicity of $K_{\Delta^{r+1}}(f, u)_{B}$ and of $E_{u}(f)_{B}$, one can obtain the following equivalent form of (4.8) and (4.9), which may appear more traditional:

$$
K_{\Delta^{r}}\left(f, t^{r}\right)_{B} \geq C t^{r}\left\{\int_{t}^{\infty} u^{-r s} K_{\Delta^{r+1}}\left(f, u^{r+1}\right)_{B}^{s} \frac{d u}{u}\right\}^{1 / s}
$$

and

$$
K_{\Delta^{r}}\left(f, t^{r}\right)_{B} \geq C t^{r}\left\{\int_{t^{1 / 2}}^{\infty} u^{-2 r s} E_{u^{-1}}(f)_{B}^{s} \frac{d u}{u}\right\}^{1 / s} .
$$

For $B=L_{p}\left(\mathbb{R}^{d}\right), \quad 1<p<\infty, \quad \omega^{r}(f, t)_{p}$ and $K_{\Delta^{r}}\left(f, t^{r}\right)_{p}$ given by (1.5) and (4.7) respectively satisfy $\omega^{2 r}(f, t)_{p} \approx K_{\Delta^{r}}\left(f, t^{2 r}\right)_{p}$ and hence (4.8) can take the form

$$
\omega^{2 r}(f, t)_{p} \geq C t^{2 r}\left\{\int_{t}^{\infty} u^{-2 r s} \omega^{2 r+2}(f, u)_{p}^{s} \frac{d u}{u}\right\}^{1 / s}, \quad s=\max (p, 2) .
$$

In fact, the result of Theorem 4.2 is given as an example of use of Theorem 4.1, and the same method can be used for many semigroups that are given by positive convolution operators on $\mathbb{R}^{d}$ or $\mathbb{T}^{d}, \quad d=1,2, \ldots$

In the next section we will give applications relating to holomorphic semigroups generated by multipliers.

\section{Cesàro summability and holomorphic semigroups}

For the purpose of this section, $H_{k}$ are eigenspaces of a self-adjoint operator $P(D)$, and $\lambda_{k}$ the eigenvalues of $P(D)$, satisfy $0 \leq \lambda_{k}, \quad \lambda_{k}<\lambda_{k+1}$. Furthermore, for our space $B$ we assume that $H_{k} \subset B, \quad H_{k} \subset B^{*}$ and that span $\left(\cup H_{k}\right)$ is dense in $B$. The expansion of $f$ is given by

$$
f \sim \sum_{k=0}^{\infty} P_{k} f
$$

where $P_{k} f$ is the projection of $f$ on $H_{k}$ in the $L_{2}$ sense (see [Di,98, (2.2)]). It was shown in $\mathrm{Da}-\mathrm{Di}, 05$ that if the Cesàro summability of some order $\ell$ is a contraction in $B$, that is

$$
\left\|C_{n}^{\ell} f\right\|_{B} \leq\|f\|_{B}
$$


for

$$
C_{n}^{\ell} f \equiv \frac{1}{A_{n}^{\ell}} \sum_{k=0}^{\infty} A_{n-k}^{\ell} P_{k} f \quad \text { where } \quad A_{m}^{\ell} \equiv \frac{(m+\ell) !}{\ell ! m !},
$$

then $T(t) f$ given by

$$
T(t) f=\sum_{k=0}^{\infty} e^{-k t} P_{k} f \quad \text { for } \quad t>0 \quad \text { and } \quad T(0) f=f
$$

is a holomorphic $C_{0}$ semigroup of contractions with its infinitesimal generator given by

$$
\mathcal{A} f \sim \sum_{k=1}^{\infty}-k P_{k} f, \quad \mathcal{D}(\mathcal{A})=\left\{f \in B: \exists g \in B \text { such that } P_{k} g=-k P_{k} f \quad \text { for all } k\right\} .
$$

The following theorem will establish among other facts that the positivity of $C_{n}^{\ell} f$ implies that it is a contraction in Orlicz spaces with the Luxemburg norm as well as with the Orlicz norm. We remind the reader that if an operator is a contraction on a space with respect to a given norm, it does not imply that it is a contraction with an equivalent norm.

Theorem 5.1. Suppose $O f(x)$ is given by

$$
O f(x)=\int_{\Omega} f(y) G(x, y) w(y) d y
$$

where $G(x, y)=G(y, x) \geq 0, \quad w(y) \geq 0$ and $\int_{\Omega} G(x, y) w(y) d y=1$. Then $O f$ is a contraction with respect to the Luxemburg norm given by

$$
\|f\|_{O_{L}(\Phi)}=\inf \left\{a \in \mathbb{R}_{+}: \int_{\Omega} \Phi\left(\frac{|f(x)|}{a}\right) w(x) d x \leq 1\right\}
$$

and with respect to the Orlicz norm given by

$$
\|f\|_{O(\Phi)}=\sup \left\{\int_{\Omega}|f(x) g(x)| w(x) d x: \int_{\Omega} \Psi(|g(x)|) w(x) d x \leq 1\right\}
$$

where $\Phi$ and $\Psi$ are associate Young functions. 
Proof. For $a \in \mathbb{R}_{+}$, which is close to the infimum in (5.6), we write

$$
\int_{\Omega} \Phi\left(\left|\frac{1}{a} O f(x)\right|\right) w(x) d x \leq \int_{\Omega} \Phi\left(\frac{1}{a} \int_{\Omega}|f(y)| G(x, y) w(y) d y\right) w(x) d x \equiv I .
$$

Using Jensen's inequality, the convexity of $\Phi$ and $\int G(x, y) w(y) d y=1$, we have

$$
\begin{aligned}
I & \leq \int_{\Omega} \int_{\Omega} \Phi\left(\frac{|f(y)|}{a}\right) G(x, y) w(y) d y w(x) d x \\
& =\int_{\Omega} \Phi\left(\frac{|f(y)|}{a}\right) w(y) d y \int_{\Omega} G(x, y) w(x) d x \\
& =\int_{\Omega} \Phi\left(\frac{|f(y)|}{a}\right) w(y) d y,
\end{aligned}
$$

which completes the proof for the Luxemburg norm of the Orlicz space. We now write

$$
\begin{aligned}
\int_{\Omega}|O f(x)||g(x)| w(x) d x & \leq \int_{\Omega}|g(x)| \int_{\Omega} G(x, y)|f(y)| w(y) d y w(x) d x \\
& =\int_{\Omega}|f(y)| w(y) \int_{\Omega}|g(x)| G(x, y) w(x) d x d y .
\end{aligned}
$$

As $\Psi$ is also a Young function and is convex, we have

$$
\int_{\Omega} \Psi\left\{\int_{\Omega}|g(x)| G(x, y) w(x) d x\right\} w(y) d y \leq \int_{\Omega} \Psi(|g(y)|) w(y) d y \leq 1,
$$

and hence our result follows.

For $L_{p}(\Omega)$ with weight $w(x) \geq 0$ the proof is easier as it follows directly from Hölder's inequality, but the result for $L_{p}$ is included in the more intricate proof of Theorem 5.1.

Clearly, the positivity of the Cesàro summability in the above context implies that

$$
C_{n}^{\ell} f(x)=\int_{\Omega} f(y) G_{n, \ell}(x, y) w(y) d y
$$

where $G_{n, \ell}(x, y)=G_{n, \ell}(y, x), \quad G_{n, \ell}(x, y) \geq 0, \quad w(y) \geq 0$, and when $1 \in H_{0}$, also $\int G_{n, \ell}(x, y) w(y) d y=1$. 
Theorem 5.2. Suppose $H_{k}, \lambda_{k}$ and $P_{k} f$ are as described at the beginning of this section, $B$ is an Orlicz space which satisfies (1.2) (for some $s, 2 \leq s<\infty$ ) with a Luxemburg norm or Orlicz norm, $C_{n}^{\ell}$ is positive for some $\ell, 1 \in H_{0}$ and $\lambda_{k}$ is a polynomial in $k$ of degree b. Then

$$
\begin{gathered}
K_{\mathcal{A}^{r}}\left(f, t^{r}\right)_{B} \approx \inf \left\{\|f-g\|_{B}+t^{r}\left\|P(D)^{r / b} g\right\|_{B}: g \in \mathcal{D}\left(P(D)^{r / b}\right)\right\} \\
K_{\mathcal{A}^{r}}\left(f, t^{r}\right)_{B} \geq C\left\{\sum_{j=1}^{\infty} 2^{-j r s} K_{\mathcal{A}^{r+1}}\left(f, t^{r+1} 2^{j(r+1)}\right)_{B}^{s}\right\}^{1 / s}
\end{gathered}
$$

and

$$
K_{\mathcal{A}^{r}}\left(f, 2^{-n r}\right)_{B} \geq C\left\{\sum_{j=1}^{n} 2^{-j r s} E_{2^{n-j}}(f)_{B}^{s}\right\}^{1 / s}
$$

where

$$
E_{n}(f)=\inf \left\{\|f-\varphi\|_{B}: \varphi \in \operatorname{span} \bigcup_{k=0}^{n} H_{k}\right\} .
$$

Proof. The proof of (5.8) follows the proof in [Da-Di,07, Th.4.3, p.83] where the result is proved for $L_{p}$ spaces. In fact, the same proof works for Banach spaces $B$ for which some order of the Cesàro summability is bounded, which implies the realization result (see Di,98, Th.6.2 and Th.7.1], and that result is the key ingredient for the proof in [Da-Di,07, Th.4.3]. We now show

$$
E_{n}(f)_{B} \leq C \inf \left(\|f-g\|_{B}+n^{-r-1}\left\|P(D)^{(r+1) / b} g\right\|_{B}\right) \approx K_{\mathcal{A}^{r+1}}\left(f, n^{-r-1}\right)_{B}
$$

The first inequality of (5.11) follows from [Di,98, Th.4.1] when we recall that $\lambda_{k} \geq 0$, and $\lambda_{k} \approx k^{b}$, (essentially $P(D), \quad(r+1) / b$ and $n^{b}$ are $-P(D), \quad \alpha m$ and $\lambda$ respectively in [Di,98). The second equivalence is treated in detail in [Da-Di,07, Section 4]. Using (5.11), we may deduce (5.10) from (5.9), which in turn is a direct application of (4.5). 
Remark 5.3. Similar to what we stated in Remark 4.4, one can give a different form of (5.9) and (5.10). For example, we have

$$
K_{\mathcal{A}^{r}}\left(f, t^{r}\right)_{B} \geq C t^{r}\left\{\sum_{n=1}^{[1 / t]} n^{-r s-1} E_{n}(f)_{B}^{s}\right\}^{1 / s} .
$$

\section{Sharp Jackson theorem for polynomials on a simplex}

For the simplex $S \in \mathbb{R}^{d}$

$$
S=\left\{\boldsymbol{x}=\left(x_{1}, \ldots, x_{d}\right): x_{i} \geq 0 \quad x_{0}=1-\sum_{i=1}^{d} x_{i} \geq 0\right\},
$$

the Jacobi weight is given by

$$
W_{\boldsymbol{\alpha}}(\boldsymbol{x})=x_{0}^{\alpha_{0}} \ldots x_{d}^{\alpha_{d}}, \quad \boldsymbol{\alpha}=\left(\alpha_{0}, \ldots, \alpha_{d}\right), \quad \alpha_{i}>-\frac{1}{2} .
$$

The self-adjoint differential operator (see [Di,95, p.226]) on $S$ with weight $w_{\boldsymbol{\alpha}}(\boldsymbol{x})$ is given by

$$
P_{\boldsymbol{\alpha}}(D)=-\sum_{\boldsymbol{\xi} \in E_{S}} w_{\boldsymbol{\alpha}}(\boldsymbol{x})^{-1} \frac{\partial}{\partial \boldsymbol{\xi}} \widetilde{d}(\boldsymbol{\xi}, \boldsymbol{x}) w_{\boldsymbol{\alpha}}(\boldsymbol{x}) \frac{\partial}{\partial \boldsymbol{\xi}} \equiv \sum_{\boldsymbol{\xi} \in E_{S}} P_{\boldsymbol{\alpha}, \boldsymbol{\xi}}(D)
$$

where $E_{S}$ is the set of directions parallel to the edges of $S$, and $\widetilde{d}(\boldsymbol{\xi}, \boldsymbol{x})$ is given by

$$
\tilde{d}(\boldsymbol{\xi}, \boldsymbol{x})=\sup _{\substack{\lambda \geq 0 \\ \boldsymbol{x}+\lambda \boldsymbol{\xi} \in S}} d(\boldsymbol{x}, \boldsymbol{x}+\lambda \boldsymbol{\xi}) \sup _{\substack{\lambda \geq 0 \\ \boldsymbol{x}-\lambda \boldsymbol{\xi} \in S}} d(\boldsymbol{x}, \boldsymbol{x}-\lambda \boldsymbol{\xi})
$$

using the Euclidean distance $d(\boldsymbol{x}, \boldsymbol{y})$.

For $\Pi_{k}$ the polynomials of total degree $\leq k$ we have $\Pi_{k}=H_{0} \oplus \cdots \oplus H_{k}$ where

$$
P_{\boldsymbol{\alpha}}(D) \varphi=\ell\left(\ell+d+\sum_{i=0}^{d} \alpha_{i}\right) \varphi \equiv \lambda_{\ell} \varphi, \quad \varphi \in H_{\ell} .
$$

Defining the $K$-functional on $S$ by

$$
K_{r}\left(f, P_{\boldsymbol{\alpha}}(D)^{r / 2}, t^{r}\right)_{B}=\inf \left(\|f-g\|_{B}+t^{r}\left\|P_{\boldsymbol{\alpha}}(D)^{r / 2} g\right\|_{B}: g \in \mathcal{D}\left(P_{\boldsymbol{\alpha}}(D)^{r / 2}\right)\right)
$$


where for $\beta \in[0, \infty) \quad P_{\boldsymbol{\alpha}}(D)^{\beta}$ is given for $\beta>0$ by

$$
P_{\boldsymbol{\alpha}}(D)^{\beta} f=\sum_{\ell=1}^{\infty} \lambda_{\ell}^{\beta} P_{\ell} f, \quad f \in \mathcal{D}\left(P_{\boldsymbol{\alpha}}(D)^{\beta}\right) \quad \text { if } \quad \exists \psi \in B \quad P_{\ell} \psi=\lambda_{\ell}^{\beta} P_{\ell} f
$$

where $P_{\ell} \varphi$ is the $L_{2}$ projection of $\varphi$ onto $H_{\ell}$. We can now deduce the sharp Jackson inequality for polynomials and lower estimate for $K$-functionals on the simplex.

Theorem 6.1. Suppose $B$ is a weighted $L_{p}$ or an Orlicz space on the simplex $S$ satisfying (1.2) for some $2 \leq s<\infty$. Then

$$
K_{r}\left(f, P_{\boldsymbol{\alpha}}(D)^{r / 2}, t^{r}\right)_{B} \geq C\left\{\sum_{j=1}^{\infty} 2^{-j r s} K_{r+1}\left(f, P_{\boldsymbol{\alpha}}(D)^{(r+1) / 2}, t^{r+1} 2^{j(r+1)}\right)_{B}^{s}\right\}^{1 / s}
$$

and

$$
K_{r}\left(f, P_{\boldsymbol{\alpha}}(D)^{r / 2}, 2^{-n r}\right)_{B} \geq C_{1} 2^{-n r}\left\{\sum_{j=0}^{n} 2^{j r s} E_{2^{j}}(f)_{B}^{s}\right\}^{1 / s}
$$

where $S, P(D), K_{r}\left(f, P_{\boldsymbol{\alpha}}(D)^{r / 2}, t\right)_{B}$ and $P_{\boldsymbol{\alpha}}(D)^{r / 2}$ are given by (6.1), (6.3), (6.6) and (6.7) respectively and $E_{n}(f)_{B}$ is given by

$$
E_{n}(f)_{B}=\inf \left(\|f-P\|_{B}: P \in \Pi_{n}\right) .
$$

Proof. We follow [Du-Xu, Cor.7.4.2, p.273], which implies the positivity of the Cesàro summability $C_{n}^{\delta}$, provided that $\delta$ is large enough. The use of Theorem 5.2 will complete the proof of (6.8), when we recall that $\lambda_{\ell}=\ell\left(\ell+d+\sum_{i=0}^{d} \alpha_{i}\right)$ is a polynomial of degree $b=2$ in $\ell$. The proof of (6.9) follows from the boundedness of the Cesàro summability which implies (see Di,98, Th.6.1])

$$
E_{n}(f)_{B} \leq C K_{r+1}\left(f, P_{\boldsymbol{\alpha}}(D)^{(r+1) / 2}, n^{-r-1}\right)_{B}
$$

and hence (6.9) can be deduced from (6.8).

For $d=1$ and $B=L_{p}$ with Jacobi weights, Theorem 6.1 was proved in Da-Di-Ti, Th.6.1]. 


\section{Sharp Jackson inequality on the sphere}

The result of this section was proved for $L_{p}\left(S^{d-1}\right), \quad 1<p<\infty$ in [Da-Di-Ti, Th.8.1]. Here we will give an alternative proof which yields an extension to a class of Banach spaces that include many Orlicz spaces.

The Laplace-Beltrami operator $\widetilde{\Delta}$ on the unit sphere $S^{d-1}=\left\{\boldsymbol{x} \in \mathbb{R}^{d}:|\boldsymbol{x}|^{2} \equiv x_{1}^{2}+\right.$ $\left.\cdots+x_{d}^{2}=1\right\}$ is given by

$$
\widetilde{\Delta} f(x)=\Delta F(x), \quad x \in S^{d-1} \quad \text { where } F(x)=f\left(\frac{x}{|x|}\right) \text { and } \Delta=\frac{\partial^{2}}{\partial x_{1}^{2}}+\cdots+\frac{\partial^{2}}{\partial x_{d}^{2}} .
$$

The eigenspace $H_{k}$ of spherical harmonic polynomials of degree $k$ on $S^{d-1}$ is given by

$$
H_{k}=\{\varphi: \widetilde{\Delta} \varphi=-k(k+d-2) \varphi\}, \quad \lambda_{k} \equiv k(k+d-2) .
$$

For a Banach space of functions on $S^{d-1}$ the $K$-functional $\widetilde{K}_{r}\left(f,(-\widetilde{\Delta})^{r / 2}, t^{r}\right)_{B}$ is given by

$$
K_{r}\left(f,(-\widetilde{\Delta})^{r / 2}, t^{r}\right)_{B}=\inf \left\{\|f-g\|_{B}+t^{r}\left\|(-\widetilde{\Delta})^{r / 2} g\right\|_{B}: g \in \mathcal{D}\left((-\widetilde{\Delta})^{r / 2}\right)\right\}
$$

where

$$
\begin{gathered}
(-\widetilde{\Delta})^{r / 2} f \sim \sum_{\ell=1}^{\infty}(\ell(\ell+d-2))^{r / 2} P_{\ell} f \\
f \in \mathcal{D}\left((-\widetilde{\Delta})^{r / 2}\right) \quad \text { if } \quad \exists \psi \in B \quad \text { satisfying } \quad P_{\ell} \psi=\lambda_{\ell}^{r / 2} P_{\ell} f
\end{gathered}
$$

and $P_{\ell} \varphi$ is the $L_{2}$ projection of $f$ on $H_{\ell}$.

We can now state and prove the result of this section.

Theorem 7.1. Suppose that $B$ is an Orlicz space of functions on $S^{d-1}$ satisfying (1.2) for some $2 \leq s \leq \infty$, and for $\rho \in S O(d)$

$$
\|f(\rho \cdot)\|_{B}=\|f(\cdot)\|_{B}, \quad\|f(\rho \cdot)-f(\cdot)\|_{B} \rightarrow 0 \quad \text { as } \quad|\rho-I| \rightarrow 0,
$$

where $|\rho-I|=\max \left\{|\rho \boldsymbol{x}-\boldsymbol{x}|: \boldsymbol{x} \in S^{d-1}\right\}$. Then for $r=1,2, \ldots$

$$
K_{r}\left(f,(-\widetilde{\Delta})^{r / 2}, t^{r}\right)_{B} \geq C\left\{\sum_{j=1}^{\infty} 2^{-j r s} K_{r+1}\left(f,(-\widetilde{\Delta})^{(r+1) / 2}, t^{r+1} 2^{j(r+1)}\right)_{B}^{s}\right\}^{1 / s}
$$


and

$$
K_{r}\left(f,(-\widetilde{\Delta})^{r / 2}, 2^{-n r}\right)_{B} \geq C_{1} 2^{-n r}\left\{\sum_{j=0}^{n} 2^{j r s} E_{2^{j}}(f)_{B}^{s}\right\}^{1 / s}
$$

where $\widetilde{\Delta}, \quad K_{r}\left(f,(-\widetilde{\Delta})^{r / 2}, t^{r}\right)_{B}$ and $(-\widetilde{\Delta})^{r / 2}$ are given by (7.1), (7.3) and (7.4) respectively, and $E_{n}(f)_{B}$ is given by

$$
E_{n}(f)_{B}=\inf \left(\|f-P\|_{B}: P \in \operatorname{span} \bigcup_{k=0}^{n-1} H_{k}\right)
$$

with $H_{k}$ of (7.2).

We remind the reader that $S O(d)$ is the collection of $d \times d$ orthogonal matrices whose determinant equals 1 .

Proof. We first recall that the Cesàro summability of order $\ell>d-1$ is a positive operator (see for instance [Du-Xu, Cor.7.2.5,p.266]). This already implies that $C_{n}^{\ell}$ is a contraction operator on $L_{p}\left(S^{d-1}\right)$. Furthermore, the above and Di,06, Th.2.1] imply that $C_{n}^{\ell}$ is a contraction on many other Banach spaces of functions on $S^{d-1}$, including all Orlicz spaces. We now use the semigroup given in (5.3) and Theorem 5.2 to obtain (17.6) when we observe that, using the technique of Da-Di,07, Section 4], $K_{\mathcal{A}^{r}}\left(f, t^{r}\right)_{B} \approx K_{r}\left(f,(-\widetilde{\Delta})^{r / 2}, t^{r}\right)_{B}$ for that semigroup for any Banach space $B$ for which the Cesàro summability is bounded. The inequality (7.7) follows using [Di,98, Th.6.1], which is applicable here as the Cesàro summability is bounded and implies

$$
E_{2^{k}}(f)_{B} \leq C K_{r+1}\left(f,(-\widetilde{\Delta})^{(r+1) / 2}, 2^{-k(r+1)}\right)_{B} .
$$




\section{Non-holomorphic semigroups and averaged moduli of smoothness}

For a semigroup $\{T(u)\}_{u \geq 0}$ on a Banach space $B$ the averaged moduli of smoothness are given by

$$
\mathrm{w}_{T}^{r}(f, t)_{B} \equiv \frac{1}{t} \int_{0}^{t}\left\|(T(u)-I)^{r} f\right\|_{B} d u .
$$

We recall that the moduli $\omega_{T}^{r}(f, t)_{B}$ are given by

$$
\omega_{T}^{r}(f, t)_{B} \equiv \sup _{0 \leq u \leq t}\left\|(T(u)-I)^{r} f\right\|_{B}
$$

and we have the following equivalence.

Theorem 8.1. Suppose $\{T(u)\}_{u \geq 0}$ is a $C_{0}$ semigroup of contractions on a Banach space B. Then

$$
\mathrm{w}_{T}^{r}(f, t)_{B} \leq \omega_{T}^{r}(f, t)_{B} \leq C(r) \mathrm{w}_{T}^{r}(f, t)_{B}
$$

Proof. We now follow verbatim the proof in [De-Lo, p.184-185]. In [De-Lo] the result refers only to $L_{p}$ and translations, but the proof is the same and the identity (5.3) in [De-Lo, p.184] is replaced by the identity

$$
(T(h)-I)^{r}=\sum_{k=1}^{r}(-1)^{k}\left(\begin{array}{l}
r \\
k
\end{array}\right)\left\{T(k h)(T(k s)-I)^{r}-(T(h+k s)-I)^{r}\right\},
$$

the proof of which is the same.

As a corollary, we obtain the following result.

Theorem 8.2. Suppose $\{T(u)\}_{u \geq 0}$ is a $C_{0}$ semigroup of contractions on a Banach space $B$ which satisfies (1.2) for some $2 \leq s<\infty$. Then

$$
\omega_{T}^{r}(f, t)_{B} \geq C\left\{\sum_{j=1}^{\infty} 2^{-j r s} \omega_{T}^{r+1}\left(f, 2^{j} t\right)_{B}^{s}\right\}^{1 / s} .
$$


Proof. Using the definition of $\omega_{T}^{r}(f, t)_{B}$, we have

$$
\omega_{T}^{r}(f, t)_{B}^{s} \geq \frac{1}{t} \int_{0}^{t}\left\|(T(u)-I)^{r} f\right\|_{B}^{s} d u .
$$

Theorem 2.1 now implies

$$
\omega_{T}^{r}(f, t)_{B}^{s} \geq \frac{m_{1}}{t} \int_{0}^{t} \sum_{j=0}^{\ell} 2^{-r s j}\left\|\left(T\left(2^{j} u\right)-I\right)^{r+1} f\right\|_{B}^{s} d u
$$

(which setting $v=2^{j} u$ )

$$
\begin{aligned}
& =m_{1} \sum_{j=0}^{\ell} 2^{-r s j} \frac{1}{2^{j} t} \int_{0}^{2^{j} t}\left\|(T(v)-I)^{r+1} f\right\|_{B}^{s} d v \\
& \geq m_{1} \sum_{j=0}^{\ell} 2^{-r s j}\left(\frac{1}{2^{j} t} \int_{0}^{2^{j} t}\left\|(T(v)-I)^{r+1} f\right\|_{B} d v\right)^{s} \\
& =m_{1} \sum_{j=0}^{\ell} 2^{-r s j} \mathrm{w}_{T}^{r+1}\left(f, 2^{j} t\right)_{B}^{s} \\
& \geq \frac{m_{1}}{(C(r+1))^{s}} \sum_{j=0}^{\ell} 2^{-r s j} \omega_{T}^{r+1}\left(f, 2^{j} t\right)_{B}^{s} .
\end{aligned}
$$

As an immediate application, we obtain the following result.

Theorem 8.3. Suppose $B$ is a Banach space of functions on $\mathbb{R}_{+}, \mathbb{R}$ or $\mathbb{T}$ satisfying (1.2) with some $2 \leq s<\infty$ and $\|f(\cdot+\xi)\|_{B} \leq\|f(\cdot)\|_{B}$ for $\xi \geq 0$. Then

$$
\omega^{r}(f, t)_{B} \geq C\left\{\sum_{j=1}^{\infty} 2^{-j r s} \omega^{r+1}\left(f, 2^{j} t\right)_{B}^{s}\right\}^{1 / s}
$$

where $\omega^{k}(f, t)_{B}$ is $\omega_{T}^{k}(f, t)_{B}$ with $T(u) f(x)=f(x+u)$.

We remark that for $\mathbb{R}_{+}$, Theorem 8.3 was not deduced in [Da-Di-Ti] even for $L_{p}\left(\mathbb{R}_{+}\right)$, with $1<p<\infty$. Of course (8.6) is valid for other spaces, not just $L_{p}$. 


\section{Results for spaces of functions on $\mathbb{R}^{d}$ or $\mathbb{T}^{d}, d>1$}

For $d>1$ we use a result on averaged moduli that stems from the work Da-Di,04 which is different from the averaged moduli in Section 8 .

We define

$$
V_{t} f(x)=\frac{1}{m_{t}} \int_{|x-y|=t} f(y) d y, \quad V_{t} 1=1
$$

where $|x-y|$ is the Euclidean distance between $x$ and $y$ for which we have the following result.

Theorem 9.1. Suppose $B$ is a Banach space of functions on $\mathbb{R}^{d}$ or $\mathbb{T}^{d}$ with $d>1$ which satisfies (1.3). Then

$$
\left\|V_{\ell, t} f-f\right\|_{B} \approx \inf _{g}\left(\|f-g\|_{B}+t^{2 \ell}\left\|\Delta^{\ell} g\right\|_{B}\right) \equiv K_{\Delta^{\ell}}\left(f, t^{2 \ell}\right)_{B}
$$

where

$$
V_{\ell, t} f \equiv \frac{-2}{\left(\begin{array}{c}
2 \ell \\
\ell
\end{array}\right)} \sum_{j=1}^{\ell}(-1)^{j}\left(\begin{array}{c}
2 \ell \\
\ell-j
\end{array}\right) V_{j t} f
$$

and $\Delta f \equiv \frac{\partial^{2} f}{\partial x_{1}^{2}}+\cdots+\frac{\partial^{2} f}{\partial x_{d}^{2}}$ is the Laplacian.

Proof. For $L_{p}\left(\mathbb{R}^{d}\right) \quad 1 \leq p \leq \infty$ and $d>1$, Theorem 9.1 was proved in Da-Di,04, Th.3.1, pp.273-276], and in fact all we do here is show how to deduce our theorem from [Da-Di,04, Th.3.1]. We note that (9.2) for $L_{\infty}\left(\mathbb{R}^{d}\right)$ implies the validity of (9.2) for $C\left(\mathbb{R}^{d}\right)$. (Perhaps the only interesting situation of (9.2) in case $B=L_{\infty}\left(\mathbb{R}^{d}\right)$ is when $B=C\left(\mathbb{R}^{d}\right)$, because only when $f \in C\left(\mathbb{R}^{d}\right)$ do both sides of (9.2) tend to zero as $t \rightarrow 0$.)

Using [Be-Da-Di, Th.6.2,p.97] with $m_{1}>\frac{2(d+2)}{d-1} \ell$, we have

$$
\left\|\Delta^{\ell} V_{k t}^{m_{1}} F\right\|_{C\left(\mathbb{R}^{d}\right)} \leq \frac{A_{1}\left(m_{1}, \ell, k\right)}{t^{2 \ell}}\|F\|_{C\left(\mathbb{R}^{d}\right)},
$$


and hence for $m$ large enough, $m>\frac{2(d+2)}{d-1} \ell^{2}$ for example, we have

$$
\left\|\Delta^{\ell} V_{\ell, t}^{m} F\right\|_{C\left(\mathbb{R}^{d}\right)} \leq \frac{A_{2}(m, \ell)}{t^{2 \ell}}\|F\|_{C\left(\mathbb{R}^{d}\right)} .
$$

We now show that for $F \in C\left(\mathbb{R}^{d}\right)$

$$
\begin{aligned}
A^{-1}\left\|F-V_{\ell, t} F\right\|_{C\left(\mathbb{R}^{d}\right)} & \leq\left\|F-V_{\ell, t}^{m} F\right\|_{C\left(\mathbb{R}^{d}\right)}+t^{2 \ell}\left\|\Delta^{\ell} V_{\ell, t}^{m} F\right\|_{C\left(\mathbb{R}^{d}\right)} \\
& \leq A\left\|F-V_{\ell, t} F\right\|_{C\left(\mathbb{R}^{d}\right)} .
\end{aligned}
$$

The left hand inequality of (9.5) is clear using (9.2) for $C\left(\mathbb{R}^{d}\right)$ (already proved in Da-Di,04, Th.3.1]), and recalling the definition of $K_{\Delta^{\ell}}\left(f, t^{2 \ell}\right)_{C\left(\mathbb{R}^{d}\right)}$. Using $\left\|V_{\ell, t} F\right\|_{C\left(\mathbb{R}^{d}\right)} \leq$ $A_{1}\|F\|_{C\left(\mathbb{R}^{d}\right)}$, we have

$$
\left\|F-V_{\ell, t}^{m} F\right\|_{C\left(\mathbb{R}^{d}\right)} \leq A_{2}\left\|F-V_{\ell, t} F\right\|_{C\left(\mathbb{R}^{d}\right)} .
$$

To conclude the proof of (9.5) we have to estimate $t^{2 \ell}\left\|\Delta^{\ell} V_{\ell, t}^{m} F\right\|_{C\left(\mathbb{R}^{d}\right)}$. We choose $G_{1}$ such that $\left\|F-G_{1}\right\|_{C\left(\mathbb{R}^{d}\right)}+t^{2 \ell}\left\|\Delta^{\ell} G_{1}\right\|_{C\left(\mathbb{R}^{d}\right)} \leq 2 K_{\Delta^{\ell}}\left(F, t^{2 \ell}\right)_{C\left(\mathbb{R}^{d}\right)}$ and write

$$
\begin{aligned}
t^{2 \ell}\left\|\Delta^{\ell} V_{\ell, t}^{m} F\right\|_{C\left(\mathbb{R}^{d}\right)} & \leq t^{2 \ell}\left\|\Delta^{\ell} V_{\ell, t}^{m}\left(F-G_{1}\right)\right\|_{C\left(\mathbb{R}^{d}\right)}+t^{2 \ell}\left\|\Delta^{\ell} V_{\ell, t}^{m} G_{1}\right\|_{C\left(\mathbb{R}^{d}\right)} \\
& \leq A_{2}(m, \ell)\left\|F-G_{1}\right\|_{C\left(\mathbb{R}^{d}\right)}+t^{2 \ell}\left\|V_{\ell, t}^{m} \Delta^{\ell} G_{1}\right\|_{C\left(\mathbb{R}^{d}\right)} \\
& \leq A_{2}(m, \ell)\left\|F-G_{1}\right\|_{C\left(\mathbb{R}^{d}\right)}+t^{2 \ell} A_{3}^{m}\left\|\Delta^{\ell} G_{1}\right\|_{C\left(\mathbb{R}^{d}\right)} \\
& \leq A_{4} K_{\Delta^{\ell}}\left(F, t^{2 \ell}\right)_{C\left(\mathbb{R}^{d}\right)} \\
& \leq A_{5}\left\|F-V_{\ell, t} F\right\|_{C\left(\mathbb{R}^{d}\right)},
\end{aligned}
$$

which concludes the proof of (9.5). To prove (9.2) for a Banach space on $\mathbb{R}^{d}$ or $\mathbb{T}^{d}$, we proceed first by showing

$$
\begin{aligned}
A^{-1}\left\|f-V_{\ell, t} f\right\|_{B} & \leq\left\|f-V_{\ell, t}^{m} f\right\|_{B}+t^{2 \ell}\left\|\Delta^{\ell} V_{\ell, t}^{m} f\right\|_{B} \\
& \leq 2 A\left\|f-V_{\ell, t} f\right\|_{B} .
\end{aligned}
$$

We first attend to Banach spaces $B$ of functions on $\mathbb{R}^{d}$. To prove the left hand inequality of (9.6), we choose $g \in B^{*}$ satisfying $\|g\|_{B^{*}}=1$ and define $F(x)=f * g(x)=\langle f(x-\cdot), g(\cdot)\rangle$. Using (1.3) we have $F \in C\left(\mathbb{R}^{d}\right)$ and recalling (9.5), we have

$$
A^{-1}\left\|F-V_{\ell, t} F\right\|_{C\left(\mathbb{R}^{d}\right)} \leq\left\|F-V_{\ell, t}^{m} F\right\|_{C\left(\mathbb{R}^{d}\right)}+t^{2 \ell}\left\|\Delta^{\ell} V_{\ell, t} F\right\|_{C\left(\mathbb{R}^{d}\right)}
$$


(so using $\|g\|_{B^{*}}=1$ and the convolution structure of $V_{\ell, t}$ will imply)

$$
\leq\left\|f-V_{\ell, t}^{m} f\right\|_{B}+t^{2 \ell}\left\|\Delta^{\ell} V_{\ell, t} f\right\|_{B}
$$

For appropriate $g_{\varepsilon}$ and $F=F_{\varepsilon}=f * g_{\varepsilon}$

$$
\left\|F-V_{\ell, t} F\right\|_{C\left(\mathbb{R}^{d}\right)} \geq\left|F(0)-V_{\ell, t} F(0)\right| \geq\left\|f-V_{\ell, t} f\right\|_{B}-\varepsilon
$$

and as $\varepsilon>0$ is arbitrary, the left inequality of (9.6) is proved.

We now follow the same technique to deduce from

$$
\left\|F-V_{\ell, t}^{m} F\right\|_{C\left(\mathbb{R}^{d}\right)} \leq A\left\|F-V_{\ell, t} F\right\|_{C\left(\mathbb{R}^{d}\right)} \quad \text { and } \quad t^{2 \ell}\left\|\Delta^{\ell} V_{\ell, t}^{m} F\right\|_{C\left(\mathbb{R}^{d}\right)} \leq A\left\|F-V_{\ell, t} F\right\|_{C\left(\mathbb{R}^{d}\right)}
$$

the inequalities

$$
\left\|f-V_{\ell, t}^{m} f\right\|_{B} \leq A\left\|f-V_{\ell, t} f\right\|_{B} \quad \text { and } \quad t^{2 \ell}\left\|\Delta^{\ell} V_{\ell, t}^{m} f\right\|_{B} \leq A\left\|f-V_{\ell, t} f\right\|_{B},
$$

which together with the above, imply (9.6) and hence (9.2) for a Banach space of functions on $\mathbb{R}^{d}$ satisfying (1.3).

To prove the result for a Banach space of functions satisfying (1.3) on $\mathbb{T}^{d}$, we observe that $C\left(\mathbb{T}^{d}\right) \subset C\left(\mathbb{R}^{d}\right)$ and that for $F \in C\left(\mathbb{T}^{d}\right), \quad V_{\ell, t}^{k} F \in C\left(\mathbb{T}^{d}\right)$ for all $k, \ell$ and $t$.

Moreover, (9.5) is satisfied with the norm $C\left(\mathbb{T}^{d}\right)$ replacing $C\left(\mathbb{R}^{d}\right)$, since if $G \in C\left(\mathbb{T}^{d}\right), \quad\|G\|_{C\left(\mathbb{T}^{d}\right)}=\|G\|_{C\left(\mathbb{R}^{d}\right)}$. We now use the same technique to deduce (9.6) for Banach spaces of functions on $\mathbb{T}^{d}$ from (9.5) with $C\left(\mathbb{T}^{d}\right)$ instead of $C\left(\mathbb{R}^{d}\right)$.

To show that the inequality (9.6) implies (9.2), we observe that the right hand inequality implies $\left\|f-V_{\ell, t} f\right\|_{B} \geq \frac{1}{2 A} K_{\Delta^{\ell}}\left(f, t^{2 \ell}\right)_{B}$. Choosing $g$ such that $\Delta^{\ell} g \in B$ and $\|f-g\|_{B}+t^{2 \ell}\left\|\Delta^{\ell} g\right\|_{B} \leq 2 K_{\Delta^{\ell}}\left(f, t^{2 \ell}\right)_{B}$, and using the left inequality of (9.6) , we write

$$
\begin{aligned}
A^{-1}\left\|f-V_{\ell, t} f\right\|_{B} \leq & \|f-g\|_{B}+\left\|V_{\ell, t}^{m}(f-g)\right\|_{B} \\
& \left.+t^{2 \ell} \| \Delta^{\ell} V_{\ell, t}^{m}(f-g)\right)\left\|_{B}+t^{2 \ell}\right\| \Delta^{\ell} V_{\ell, t}^{m} g \|_{B} .
\end{aligned}
$$

We now follow the method used earlier to deduce

$$
\left\|V_{\ell, t}^{m} f\right\|_{B} \leq A_{5}\|f\|_{B} \quad \text { and } \quad\left\|\Delta^{\ell} V_{\ell, t}^{m} f\right\| \leq \frac{A_{2}(m, \ell)}{t^{2 \ell}}\|f\|_{B} \quad \text { for all } \quad f \in B
$$


from the corresponding inequalities for $B=C\left(\mathbb{R}^{d}\right)$ or $B=C\left(\mathbb{T}^{d}\right)$. We also need to recall that $\left\|\Delta^{\ell} V_{\ell, t}^{m} g\right\|_{B}=\left\|V_{\ell, t}^{m} \Delta^{\ell} g\right\|_{B}$ whenever $\Delta^{\ell} g \in B$ to complete the proof.

Theorem 9.2. Suppose $B$ is a Banach space of functions on $\mathbb{R}^{d}$ or $\mathbb{T}^{d}$ and its norm satisfies (1.2) for some $s, 2 \leq s<\infty$, and (1.3). Then for any $\ell$ such that $2 \ell>r$

$$
\omega^{r}(f, t)_{B} \geq C\left\{\sum_{j=1}^{\infty} 2^{-j r s} K_{\Delta^{\ell}}\left(f,\left(2^{j} t\right)^{2 \ell}\right)_{B}^{s}\right\}^{1 / s} .
$$

Proof. We write

$$
\omega^{r}(f, t)_{B}^{s}=\sup _{|u| \leq t}\left\|\Delta_{u}^{r} f\right\|_{B}^{s} \geq \sup _{|u|=t}\left\|\Delta_{u}^{r} f\right\|_{B}^{s} \geq \frac{1}{m_{t}} \int_{|u|=t}\left\|\Delta_{u}^{r} f\right\|_{B}^{s} d u
$$

with $m_{t}$ of (9.1) i.e. $\int_{|u|=t} d u=m_{t}$. We now use Theorem 2.1 with $T=T(u)$ and $T(u) f(x)=f(x+u)$ to obtain

$$
\begin{aligned}
\omega^{r}(f, t)_{B}^{s} & \geq \frac{C}{m_{t}} \int_{|u|=t} \sum_{j=1}^{L} 2^{-j r s}\left\|\Delta_{2^{j} u}^{r+1} f\right\|_{B}^{s} d u \\
& \geq \frac{C_{1}}{m_{t}} \int_{|u|=t} \sum_{j=1}^{L} 2^{-j r s}\left\|\Delta_{2^{j} u}^{2 \ell} f\right\|_{B}^{s} d u \\
& =C_{1} \sum_{j=1}^{L} 2^{-j r s} \frac{1}{m_{t} 2^{j(d-1)}} \int_{|v|=2^{j} t}\left\|\Delta_{v}^{2 \ell} f\right\|_{B}^{s} d v .
\end{aligned}
$$

As translations are isometries (see (1.3)), we have

$$
\left\|\Delta_{v}^{2 \ell} f(\cdot)\right\|_{B}=\left\|\sum_{k=-\ell}^{\ell}(-1)^{k}\left(\begin{array}{c}
2 \ell \\
\ell-k
\end{array}\right) f(\cdot+k v)\right\|_{B} .
$$

Therefore, using the Hölder and the triangle inequality we have

$$
\omega^{r}(f, t)_{B}^{s} \geq C_{1} \sum_{j=1}^{L} 2^{-j r s}\left\|\frac{1}{m_{t} 2^{j(d-1)}} \int_{|v|=2^{j} t} \sum_{k=-\ell}^{\ell}(-1)^{k}\left(\begin{array}{c}
2 \ell \\
\ell-k
\end{array}\right) f(\cdot+k v) d v\right\|_{B}^{s} .
$$


Since $\int_{|v|=2^{j} t} d v=m_{t} 2^{j(d-1)}$, we now have (using Theorem 9.1)

$$
\begin{aligned}
\omega^{r}(f, t)_{B}^{s} & \geq C_{1} \sum_{j=1}^{L} 2^{-j r s}\left(\begin{array}{c}
2 \ell \\
\ell
\end{array}\right)\left\|V_{\ell, 2^{j}} f-f\right\|_{B}^{s} \\
& \geq C_{2} \sum_{j=1}^{L} 2^{-j r s} K_{\Delta^{\ell}}\left(f,\left(2^{j} t\right)^{2 \ell}\right)_{B}^{s} .
\end{aligned}
$$

The sharp-Jackson result can now be deduced from Theorem 9.2 .

Theorem 9.3. Suppose $B$ is a Banach space of functions on $\mathbb{R}^{d}$ or $\mathbb{T}^{d}$ satisfying (1.2) and (1.3). Then

$$
\omega^{r}(f, t)_{B} \geq C\left\{\sum_{j=1}^{\infty} 2^{-j r s} E_{1 /\left(t 2^{j}\right)}(f)_{B}^{s}\right\}^{1 / s}
$$

where $E_{\lambda}(f)_{B}$ is given in (4.10) when $B$ is a space of functions on $\mathbb{R}^{d}$ and by

$$
E_{\lambda}(f)_{B}=\inf \left\{\|f-\varphi\|_{B}: \varphi(\boldsymbol{x})=\sum_{|\boldsymbol{n}|<\lambda} a_{\boldsymbol{n}} e^{i \boldsymbol{n} \boldsymbol{x}}\right\}
$$

when $B$ is a space of functions on $\mathbb{T}^{d}$.

Proof. When $E_{\lambda}(f)_{B}$ is given by (4.10), we use (4.12) to deduce (9.4) from (9.3), writing

$$
f=f-\varphi_{1 / t}+\left(\varphi_{1 / 2 t}-\varphi_{1 / t}\right)+\cdots+\left(\varphi_{1 / 2^{i} t}-\varphi_{1 / 2^{i-1} t}\right)+\varphi_{1 / 2^{i} t}
$$

where $\varphi_{\lambda}$ is a near best approximant i.e. $\left\|f-\varphi_{\lambda}\right\|_{B} \leq a E_{\lambda}(f)_{B}$. When $E_{\lambda}$ is given by (9.9), we use the analogue of (4.12) and the same expansion to obtain (9.8).

The lower estimate of $\omega^{r}(f, t)_{B}$ is given in the following theorem.

Theorem 9.4. Under the conditions of Theorem 9.3, we have

$$
\omega^{r}(f, t)_{B}^{s} \geq C_{1} \sum_{j=1}^{L} 2^{-j r s} \omega^{r+1}\left(f, t 2^{j}\right)_{B}^{s}
$$

where $L=\min \left(\ell: 2^{-\ell} \leq t\right)$ and $B$ is a space of functions on $\mathbb{T}^{d}$ or $\mathbb{R}^{d}$. 
Proof. Since when $2^{-\ell} \leq t<2^{-\ell+1}, \quad \omega^{k}\left(f, 2^{-\ell}\right) \leq \omega^{k}(f, t)_{B} \leq \omega^{k}\left(f, 2^{-\ell+1}\right)_{B} \leq 2^{k} \omega^{k}\left(f, 2^{-\ell}\right)_{B}$, it is sufficient to prove (9.10) for $t=2^{-n}$ and $L=n$. For a Banach space of functions on $\mathbb{R}^{d}$ or $\mathbb{T}^{d}$ satisfying (1.3), the weak converse inequality yields

$$
\omega^{r+1}\left(f, 2^{-n+j}\right)_{B} \leq C_{2}\left\{\sum_{k=0}^{n-j} 2^{-k(r+1)} E_{2^{n-j-k}}(f)_{B}+\frac{1}{2^{(n-j)(r+1)}}\|f\|_{B}\right\} .
$$

Therefore, recalling $2 \leq s<\infty$, we have

$$
\begin{aligned}
\sum_{j=1}^{n} 2^{-j r s} \omega^{r+1}\left(f, 2^{-n+j}\right)_{B}^{s} \leq & C_{2}^{s}\left\{\sum_{j=1}^{n} 2^{-j r s}\left(\sum_{k=0}^{n-j} 2^{-k(r+1)} E_{2^{n-j-k}}(f)_{B}+\frac{1}{2^{(n-j)(r+1)}}\|f\|_{B}\right)^{s}\right\} \\
\leq & C_{3}\left\{\sum_{j=1}^{n} 2^{-j r s}\left(\sum_{k=0}^{n-j} 2^{-k s(r+1)} E_{2^{n-j-k}}(f)_{B}^{s}\right)\right. \\
& \left.+\sum_{j=1}^{n} 2^{-j r s} 2^{-(n-j)(r+1) s}\|f\|_{B}^{s}\right\} \\
\leq & C_{3}\left[\sum_{j=1}^{n} 2^{-j r s} \sum_{m=0}^{n-j} 2^{-(n-j-m) s(r+1)} E_{2^{m}}(f)_{B}^{s}\right]+C_{3} 2^{-n r s}\|f\|_{B}^{s} \\
= & C_{3}\left[\sum_{m=0}^{n} E_{2^{m}}(f)_{B}^{s} 2^{-(n-m) s(r+1)} \sum_{j=1}^{n-m} 2^{j s}\right]+C_{3} 2^{-n r s}\|f\|_{B}^{s} \\
\leq & C_{4} \sum_{m=1}^{n} E_{2^{m}}(f)_{B}^{s} 2^{-(n-m) s r}+C_{3} 2^{-n r s}\|f\|_{B}^{s} .
\end{aligned}
$$

In view of (9.8) (for $\left.t=2^{-n}\right)$, we have

$$
\omega^{r}(f, t)_{B}^{s}+t^{r s}\|f\|_{B}^{s} \geq C_{5} \sum_{j=1}^{L} 2^{-j r s} \omega^{r+1}\left(f, t 2^{j}\right)_{B}^{s} .
$$

We choose $g$ so that $\|f-g\|_{B}=E_{1}(f)_{B}$ where $E_{\lambda}(f)_{B}$ is given in (4.10) and (9.11) for function spaces on $\mathbb{R}^{d}$ or $\mathbb{T}^{d}$ respectively. Using (9.12), we now write

$$
\begin{aligned}
\sum_{j=1}^{L} 2^{-j r s} \omega^{r+1}\left(f-g, t 2^{j}\right)_{B}^{s} & \left.<C_{5}^{-1}\left\{\omega^{r}(f-g, t)_{B}^{s}+t^{r s} \| f-g\right) \|_{B}^{s}\right\} \\
& \leq C_{6} \omega^{r}(f, t)_{B}
\end{aligned}
$$


since $\|f-g\|_{B}+C \omega^{r}(f, 1)_{B} \leq C_{1} t^{-r} \omega^{r}(f, t)_{B} \quad$ (see $[\mathrm{Di}, 99$, Th.2.1]).

For $g \in C^{r}$ one has

$$
\omega^{r}(g, \tau)_{B} \leq \tau^{r} \max _{\xi}\left\|\left(\frac{\partial}{\partial \xi}\right)^{r} g\right\|_{B}
$$

This follows from $\left\|\Delta_{h}^{r} g\right\|_{\infty} \leq|h|^{r}\left\|\left(\frac{\partial}{\partial \xi}\right)^{r} g\right\|_{\infty}$ for $h$ in the $\xi$ direction and hence following the arguments used in Theorem 9.1 (and elsewhere), $\left\|\Delta_{h}^{r} g\right\|_{B} \leq|h|^{r}\left\|\left(\frac{\partial}{\partial \xi}\right)^{r} g\right\|_{B}$ (with $h$ still in the $\xi$ direction). Using (1.5) we now have (9.13).

Therefore, as $g \in C^{\infty}$ and $2^{-L} \approx t$, we have

$$
\begin{aligned}
\sum_{j=1}^{L} 2^{-j r s} \omega^{r+1}\left(g, t 2^{j}\right)_{B}^{s} & \leq \sum_{j=1}^{L} 2^{-j r s}\left(t 2^{j}\right)^{(r+1) s} \max _{\xi}\left\|\left(\frac{\partial}{\partial \xi}\right)^{r+1} g\right\|_{B}^{s} \\
& \leq C_{7} t^{r s} \max _{|\xi|=1}\left\|\left(\frac{\partial}{\partial \xi}\right)^{r+1} g\right\|_{B}^{s} .
\end{aligned}
$$

For function spaces on $\mathbb{T}^{d},\left\|\left(\frac{\partial}{\partial \xi}\right)^{r+1} g\right\|_{B}^{s}=0$. For function spaces on $\mathbb{R}^{d}$ we note that $\operatorname{supp} \hat{g}(y) \subset\{y:|y| \leq 1\}$ implies $\operatorname{supp} \widehat{\left(\frac{\partial}{\partial \xi}\right)^{r} g}(y) \subset\{y:|y| \leq 1\}$ and using Da-Di,04, Th. 2.1] with $R=1$ and $\ell=1$, we have

$$
\left\|\Delta\left(\frac{\partial}{\partial \xi}\right)^{r} g\right\|_{\infty} \leq C\left\|\left(\frac{\partial}{\partial \xi}\right)^{r} g\right\|_{\infty}
$$

and hence

$$
\left\|\Delta\left(\frac{\partial}{\partial \xi}\right)^{r} g\right\|_{B} \leq C\left\|\left(\frac{\partial}{\partial \xi}\right)^{r} g\right\|_{B} .
$$

We now use [Di,89, Th. 6.2] to obtain

$$
\left\|\left(\frac{\partial}{\partial \xi}\right)^{r+1} g\right\|_{B} \leq C_{8}\left\|\Delta\left(\frac{\partial}{\partial \xi}\right)^{r} g\right\|_{B}^{1 / 2}\left\|\left(\frac{\partial}{\partial \xi}\right)^{r} g\right\|_{B}^{1 / 2} \leq C_{9}\left\|\left(\frac{\partial}{\partial \xi}\right)^{r} g\right\|_{B} .
$$

Therefore,

$$
t^{r s} \max _{|\xi|=1}\left\|\left(\frac{\partial}{\partial \xi}\right)^{r+1} g\right\|_{B}^{s} \leq t^{r s} \max _{|\xi|=1}\left\|\left(\frac{\partial}{\partial \xi}\right)^{r} g\right\|_{B}^{s} \leq C_{10} t^{r s} \omega^{r}(f, 1)_{B}^{s} \leq C_{11} \omega^{r}(f, t)_{B}^{s} .
$$

We thank F. Dai for some valuable comments and for showing that the second term on the left of (9.12) is redundant not only for function spaces on $\mathbb{T}^{d}$. 


\section{References}

[Be-Da-Di] E. Belinsky, F. Dai and Z. Ditzian, Multivariate approximating averages, Jour. Approx. Theory 125 (2003), 85-105.

[Be-Sh] C. Bennet and R. Sharpley, Interpolation of Operators, Academic Press, 1988.

[Bu-Be] P.L. Butzer and H. Berens, Semi-groups of Operators and Approximation, Springer Verlag, 1967.

[Da-Di,04] F. Dai and Z. Ditzian, Combinations of multivariate averages, Jour. Approx. Theory 131 (2004), 268-283.

[Da-Di,05] F. Dai and Z. Ditzian, Strong converse inequality for Poisson sums, Proc. Amer. Math. Soc. 133 (2005), 2609-2611.

[Da-Di,07] F. Dai and Z. Ditzian, Cesàro summability and Marchaud inequality, Constr. Approx. 25 (2007), 73-88.

[Da-Di,08] F. Dai and Z. Ditzian, Jackson inequality for Banach spaces on the sphere, Acta Math. Hungar. 118 (2008), 171-195.

[Da-Di-Ti] F. Dai, Z. Ditzian and S. Tikhonov, Sharp Jackson inequalities, Jour. Approx. Theory 151 (2008), 86-112.

[De-Lo] R. DeVore and G. Lorentz, Constructive Approximation, Springer Verlag, 1993.

[Di,88] Z. Ditzian, On the Marchaud inequality, Proc. Amer. Math. Soc. 103 (1988), 198-202.

[Di,89] Z. Ditzian, Multivariate Landau-Kolmogorov-type inequality, Math. Proc. Camb. Phil. Soc. 105 (1989), 335-350.

[Di,95] Z. Ditzian, Multidimensional Jacobi-type Bernstein-Durrmeyer operators, Acta Sci. Math. (Szeged) 60 (1995), 225-243.

[Di,98] Z. Ditzian, Fractional derivatives and best approximation, Acta Math. Hungar. 81(4) (1998), 323-348.

[Di,99] Z. Ditzian, A modulus of smoothness on the unit sphere, Jour. D'Anal. Math. 79 (1999), 189-200. 
[Di,06] Z Ditzian, Approximation on Banach spaces of functions on the sphere, Jour. Approx. Theory 140 (2006), 31-45

[Di-Iv] Z. Ditzian and K.G. Ivanov, Strong converse inequalities, Jour. D’Anal. Math. 61 (1993), 61-111.

[Di-Pr] Z. Ditzian and A. Prymak, Sharp Marchaud and converse inequalities in Orlicz spaces, Proc. Amer. Math. Soc. 135 (2007), 1115-1121.

[Du-Xu] C.F. Dunkl and Y. Xu, Orthogonal Polynomials of Several Variables, Cambridge University Press, 2001.

[Li-Tz] Y. Lindenstrauss and L. Tzafriri, Banach Spaces, Vol. II, Springer-Verlag, 1979.

[Ra-Re] M.M. Rao and Z.D. Ren, Theory of Orlicz Spaces, Marcel Dekker, 1991.

[To] V. Totik, Sharp converse theorem of $L^{p}$ polynomial approximation, Constr. Approx. 4 (1988), 419-433. 